\title{
A Linear Specification Language for Petri nets
}

\author{
Carolyn Brown Doug Gurr \\ University of Aarhus*
}

\author{
Valeria de Paiva \\ University of Cambridge
}

October 1991

\begin{abstract}
This paper defines a category GNet with object set all Petri nets. A morphism in GNet from a net $\mathrm{N}$ to a net $\mathrm{N}^{\prime}$ gives a precise way of simulating every evolution of $\mathrm{N}$ by an evolution of $\mathrm{N}^{\prime}$. We exhibit a morphism from a simple message hanker to one with error-correction, showing that the more refined message handler can simulate any behaviour of its simple counterpart. The existence of such a morphism proves the correctness of the refinement.

Earlier work [Bro90, BG90, BG] defined a modular theory of elementary Petri nets based on de Paiva's Dialectica categorical models of linear logic. We here modify her construction, defining categories $\mathbf{M}_{N} \mathbf{C}$ which model intutionistic linear logic [GL87]. GNet arises naturally from $\mathbf{M}_{\mathbb{N}}$ Set inheriting the structure which models linear logic. This more general framework has several advantages over our previous one. The theory is simplified, we obtain precise results about morphisms as simulations, relating them to CCS, and we obtain a natural extension to marked nets.

The linear connectives are modelled in GNet by net combinators. Being functorial, these combinators $o p_{i}$ are such that, if each $\mathrm{N}_{j}$ is refined by a net $\mathrm{N}_{j}^{\prime}$, then $o p_{i}\left(\mathrm{~N}_{0}, \ldots, \mathrm{N}_{m}\right)$ is refined by $o p_{i}\left(\mathrm{~N}_{0}^{\prime}, \ldots, \mathrm{N}_{m}^{\prime}\right)$. We show that the operation of restriction also has this property, and thus (in the language of algebraic specification) our notion of refinement composes horizontally with respect to the linear connectives and
\end{abstract}

${ }^{*}$ Both C. Brown and D. Gum are supported by BRA CEDISYS 
restriction. Furthermore, our notion of refinement composes vertically because it corresponds to categorical morphisms. These properties of our notion of refinement are precisely those required to develop an algebra of nets in which complex nets can be bit from smaller components, and refined in a modular and compositional way. We illustrate our approach with an extended example, analogous to Milner's Jobshop example.

\section{Introduction}

Petri nets [Rei85] are a long established and relatively successful model of concurrent systems. They admit an appealing operational interpretation, the "Token Game", and their simple graphical presentation makes them a convenient system with which to describe or specify concurrent systems. This is evidenced by the successful industrial application of systems such as Jensen's Petri Net Tool [Jen90]. Finally, they provide a framework in which to investigate the issues relating to non-interleaving models of concurrency.

Unfortunately, there are significant difficulties in using Petri nets to describe and specify concurrent systems, primarily the lack of good notions of refinement and modularity. By refinement we mean the process of building an increasingly detailed description of a system by progressively replacing simple components with more complex ones. Intuitively, a net $\mathrm{N}^{\prime}$ refines $\mathrm{N}$ if $\mathrm{N}^{\prime}$ incorporates more design decisions than $\mathrm{N}$ : in this case we expect $\mathrm{N}^{\prime}$ to be able to simulate every evolution of $\mathrm{N}$, in the sense that every evolution of $\mathrm{N}$ induces a corresponding evolution in $\mathrm{N}^{\prime}$. Modularity allows us to build complex systems from simpler component subsystems. These ideas, which correspond respectively to the notions of vertical and horizontal composition of refinements in the field of algebraic specification [Wir71, ST88], are essential to developing an implementable theory of specifications (in the manner of $Z$ [AS79], Clear [BG80] and VDM [Jon86]). We expect suitable notions of refinement and modularity to satisfy certain properties. Firstly, we expect the identity operation to be a refinement. Secondly, if we have a refinement of a net $\mathrm{N}_{0}$ by $\mathrm{N}_{1}$ and a refinement of the net $\mathrm{N}_{1}$ by $\mathrm{N}_{2}$ then it should be possible to compose these to obtain a refinement of $\mathrm{N}_{0}$ by $\mathrm{N}_{2}$. Thirdly, we would like a number of net building operations $o p_{i}$ with which to construct a complex net $o p_{i}\left(\mathrm{~N}_{0}, \ldots, \mathrm{N}_{n}\right)$ from any component nets $\mathrm{N}_{0}, \ldots, \mathrm{N}_{n}$. Fi- 
nally, we want our refinement to compose horizontally with respect to each of these operations $o p_{i}$ [ST88]. That is, if each $\mathrm{N}_{j}$ is refined by a net $\mathrm{N}_{j}^{\prime}$ then $o p_{i}\left(\mathrm{~N}_{0}, \ldots, \mathrm{N}_{n}\right)$ should be refined by $o p_{i}\left(\mathrm{~N}_{0}^{\prime}, \ldots, \mathrm{N}_{n}^{\prime}\right)$.

Winskel [Win84] has addressed these issues by defining a category in which the objects were Petri nets and the morphisms represented partial simulations. There are several advantages to a categorical approach. Using morphisms to represent refinements ensures that we have compositionality of refinements, and furthermore that the composition of refinements is associative. That is, if we have refinements $f: \mathrm{N}_{0} \rightarrow \mathrm{N}_{1}, g: \mathrm{N}_{1} \rightarrow \mathrm{N}_{2}$ and $h: \mathrm{N}_{2} \rightarrow \mathrm{N}_{3}$ then refining $\mathrm{N}_{0}$ to $\mathrm{N}_{2}$ by $f g$ and then refining by $h$ is equivalent to refining $\mathrm{N}_{0}$ to $\mathrm{N}_{1}$ by $f$ and then refining by $g h$. In addition, any structure that the category of nets possesses (for example, products and coproducts) yields the constructors on nets we require for a modular approach. The functoriality of the categorical constructions ensures that refinement composes horizontally with these constructors. Thus we have a basis for an algebraic theory of specification using Petri nets.

Further, categories with sufficient structure are endowed with an associated logic. Thus Cartesian closed categories correspond to simply typed lambda calculi [LS86], toposes correspond to intuitionistic logic [Fou80, Joh77] and symmetric monoidal closed categories with certain other structure appear to correspond to Girard's Linear Logic [Amb91, See89]. Therefore, if our category of nets has the appropriate structure, we obtain a logic for reasoning about nets and refinement. In addition, expressing models of concurrency as categories enables us to explore the relationships between models by exhibiting functors between the categories. Notably, Winskel and Nielson [Win84, NW91] have shown that many different models of concurrency can be related by reflections or coreflections between the associated categories. Finally, the level of generality offered by a categorical approach often makes it relatively straightforward to modify the structures under consideration.

We have described a number of advantages of the categorical approach. However, these pleasant algebraic properties are to no avail if the categorical structures do not have a meaningful computational interpretation. In particular, the definition of morphism between nets should accord with the intuitive notion of refinement, and the categorical constructions on nets should correspond to useful net-building operations. The difficulties in defining a suitable notion of net morphism are demonstrated by the large number of 
categories of nets which has been proposed [Bro90, BG90, BG, DMM89, MM88a, MOM89, NRT90, Win87, Win88]. Many of these categories arise as instances or as subcategories of instances of the construction we now present.

In this paper, we define a new category of Petri nets based on de Paiva's categorical models of Girard's linear logic [Gir87]. The morphisms arising from this abstract approach have an appealing computational interpretation in terms of simulations. Further, for a wide class of nets, all simulations are captured by our morphisms. Thus we obtain a compositional notion of net refinement. Our category is a sound model of linear logic and so has a rich categorical structure. In particular, it is symmetric monoidal closed and has finite products and coproducts. These constructions, together with restriction (net containment) constitute the operations we require to give a modular theory of nets. Our notion of refinement composes horizontally with respect to each of these operations. We here apply this theory in a detailed example along the lines of Milner's Jobshop [Mil89]. Also, we obtain a natural extension from unmarked nets to marked nets. This preserves the categorical structure required for a modular theory of net specification, and retains the interpretation of morphisms as simulation. Since our category is a model of linear logic, we can develop a linear proof system for reasoning about net refinement.

This paper contains three important extensions of our previous work [Bro90, BG90, BG]. We extend our construction from elementary Petri nets (nets in which no arc has weight greater than 1) to all Petri nets. We obtain results giving a precise understanding of the morphisms as simulations which are closely analogous to simulation in labelled transition systems, and we give a more detailed analysis of the ideas of simulation and modularity. Our model encompasses several others. Further, it admits a natural extension to marked nets which preserves the categorical structure required for a modular theory of net specification, and retains the interpretation of morphisms as simulation.

\section{Summary of the Paper}

In this paper we address the issue of modular specification of concurrent processes by constructing categories of Petri nets. In Section 3 we review the definitions and properties of Petri nets. Recall [DP89, DP88] that de 
Paiva constructs a class of categories GC, in which objects are relations in a category $\mathbf{C}$ and the morphisms give a notion of map between relations. In [Bro90, BG90, BG] we constructed a category of elementary Petri nets based on GC. In Section 4 we modify the construction of GC to obtain a class of categories $\mathbf{M}_{\mathbb{N}} \mathbf{C}$ whose objects are generalised relations. In Section 5 we show that the categories $\mathbf{M}_{\mathbb{N}} \mathbf{C}$, like the categories $\mathbf{G C}$, have sufficient structure to model Girard's linear logic [Gir87].

In Section 6 we construct a category GNet of general nets, based on the category $\mathbf{M}_{\mathbb{N}}$ Set. In our earlier work we indicated how the morphisms could be understood as a notion of refinement or simulation. In Section 6 we make this precise. We show that whenever there is a morphism $\langle f, F\rangle$ from $\mathrm{N}$ to $\mathrm{N}^{\prime}$ in GNet, if $\mathrm{N}$ can evolve under a sequence of events $e_{0}, e_{1}, \ldots, e_{n}$ then $\mathrm{N}^{\prime}$ can evolve under the sequence of events $f\left(e_{0}\right), f\left(e_{1}\right), \ldots, f\left(e_{n}\right)$. We also relate this to the notion of simulation in labelled transition systems such as CCS. Finally, we give a practical example of a morphism which shows how a simple message handler is simulated by a more sophisticated one.

In Section 7 we characterise GNet as a limit in Cat. As a consequence, all the structure of $\mathbf{M}_{\mathbb{N}}$ Set lifts to $\mathbf{G N e t}$, which has finite products, finite coproducts and is symmetric monoidal closed. Thus GNet has sufficient structure to model intuitionistic linear logic. We study in detail the product and coproduct of two nets. In particular, we show how to represent both the synchronous product of two nets and a restricted product which allows specified asynchronous events. We prove that the behaviour of the product of two nets is the product of the behaviours of its component nets.

In Section 8 we develop our compositional theory of nets. We first give a simple condition on restrictions of a product net which ensures that refinement composes horizontally with restriction. We illustrate our theory using a detailed worked example related to Milner's Jobshop [Mil89], which builds a large net in a modular way from smaller component nets. We illustrate the horizontal composition of our refinement with respect to our constructors in the following way. We refine the "Jobber" component to introduce a distinction between hard and easy jobs, and show that there is a morphism from the old jobber to the new one and hence that the new jobber simulates the old jobber. The naturality of our constructions ensures that there is a morphism from the the old compound net to the new compound net, and hence that the new compound net simulates the old one. 
In Section 9, we sketch the application of linear logic proof terms to reasoning about net refinements. This is work in progress.

In the main part of this paper, as in [Bro90, BG90, BG], we have considered nets without initial markings. In Section 10 we show how the results of Section 6 enable us to extend naturally to a category MNet of marked nets. There is a forgetful functor $\mathcal{U}$ from MNet to GNet which has both left and right adjoints. Thus $\mathcal{U}$ preserves any small lists and colimits that exist in MNet. We prove that MNet, has finite products and finite coproducts, describe the product and coproduct of two marked nets and discuss the monoidal closed structure.

\section{Preliminary Definitions for Petri Nets}

Petri nets model processes by indicating the changes in local states (conditions) which are induced by the occurrence of events. The causal dependency between conditions and events is expressed using two multirelations: the precondition relation indicates which conditions must be satisfied before an event can occur, while the post-condition relation indicates the conditions resulting from the occurrence of an event. These two multirelations determine the dynamic behaviour of a net.

An introduction to Petri nets is given in [Rei85]. In defining Petri nets and their behaviour we assume standard definitions concerning multirelations and multisets [Win88].

Definition 3.1 $A$ Petri Net is a 4-tuple $\langle E, B$, pre, post $\rangle$, where $E$ and $B$ are sets, and pre and post are functions from $E \times B$ to $\mathbb{N}$ (multirelations).

We shall call elements of $E$ events and elements of $B$ conditions. We shall call pre and post the pre- and post-condition relations of $\mathrm{N}$ respectively. We write $\mathrm{N}$ for the Petri net $\langle E, B$, pre, post $\rangle, \mathrm{N}_{0}$ for the net $\left\langle E_{0}, B_{0}\right.$, pre $_{0}$, post $\left._{0}\right\rangle$ and so on. We write Petri for the class of Petri nets. A net $\mathrm{N}$ is elementary if the images of both pre and post are contained in $\mathbf{2}$.

With each of the multirelations pre and post: $E \times B \rightarrow \mathbb{N}$, we associate a function with the same name, from $E$ to multisets over $B$, defined by the 
formal sums

$$
\operatorname{pre}(e)=\sum_{b \in B} \operatorname{pre}\langle e, b\rangle b \text { and } \operatorname{post}(e)=\sum_{b \in B} \operatorname{post}\langle e, b\rangle b .
$$

We call pre(e) the pre-condition set of $e$, and post(e) the post-condition set of $e$. We extend the function pre (and similarly post) to a multiset of events $A: E \rightarrow \mathbb{N}$ as follows:

$$
\operatorname{pre}(A)=\sum_{e \in E} A(e) \operatorname{pre}(e) \text { for any multiset } A \text { over } E
$$

Definition 3.2 Let $\mathrm{N}$ be a Petri net. A marking of $\mathrm{N}$ is a function $M: B \rightarrow$ $\mathbb{N}$ (a multiset over $B)$. We write Mark $(\mathrm{N})$ for the set of all markings of $\mathrm{N}$. $A$ marked net is a pair $\langle\mathrm{N}, M\rangle$, where $\mathrm{N}$ is a Petri net and $M$ is a marking of $\mathrm{N}$.

Remark 3.3 Various authors add further conditions to the definition of a net, or of its markings, to ensure convergence of the relevant formal sums. It suffices to require that every event of the net has unite pre- and postcondition set, and that every condition is in the post-condition set of finitely many events.

There is a graphical representation of Petri nets in which events are represented by labelled boxes, conditions by labelled circles, and the pre- and post-condition relations by weighted, directed arcs. We shall omit weights of value 1 .

\subsection{The Evolution of Petri Nets}

We call the dynamic behaviour of a Petri net its evolution. The evolution of a net $\mathrm{N}$ reflects the causal dependencies of the process which $\mathrm{N}$ models, since the pre- and post-condition relations express causal dependency. Events which are causally independent may occur concurrently.

Let $\langle\mathrm{N}, M\rangle$ be a marked Petri net and let $A$ be a multiset over $E$. We say $>\mathrm{N}, M\left\langle\right.$ enables $A$, written $M \downarrow^{\mathrm{N}} A$, if for each $\left.b \in B, \sum_{e \in E} A(e) p r e\right\rangle e, b\langle\leq$ $M(b)$. Further, we say that $\mathrm{N}$ one-step evolves under $A$ from the marking $M$ to the marking $M^{\prime}$, written $M \sim_{1} M^{\prime}$, if

$$
M^{\prime}=(M-\operatorname{pre}(A))+\operatorname{post}(A) \text {. }
$$


In this case, the events of $A$ are said to occur concurrently, in what we shall call a transition. The derivability relation of a net $\mathrm{N}$, written $\sim$, is the transitive closure of $\sim_{1}$. We say a net $\mathrm{N}$ evolves from a marking $M$ to a marking $M^{\prime}$ if $M \leadsto M^{\prime}$. We sometimes label such an evolution, writing $M \stackrel{A}{\sim} M^{\prime}$.

\section{A Category of Multirelations}

We now extend the treatment of relations in de Paiva's dialectica category GC to multirelations, using a variation of Chu's construction [Bar79]. Our construction relates closely to the categories $\mathbf{G A M E}_{K}$ [LS91] and DecGC [DP89].

Lemma 4.1 Let $\mathrm{C}$ be a concrete ${ }^{1}$ category with finite products. Let $N$ be an object of $\mathbf{C}$, equipped with a partial order $\leq$. The following data:

- objects are triples $\langle E, B, \alpha\rangle$, where $E$ and $B$ are objects of $\mathbf{C}$ and $\alpha: E \times B \rightarrow N$ is a morphism in $\mathbf{C}$,

- a morphism from $\langle E, B, \alpha\rangle$ to $\left\langle E^{\prime}, B^{\prime}, \alpha^{\prime}\right\rangle$ is a pair $\langle f, F\rangle$ of morphisms in $\mathbf{C}$ such that $f: E \rightarrow E^{\prime}, F: B^{\prime} \rightarrow B$ and

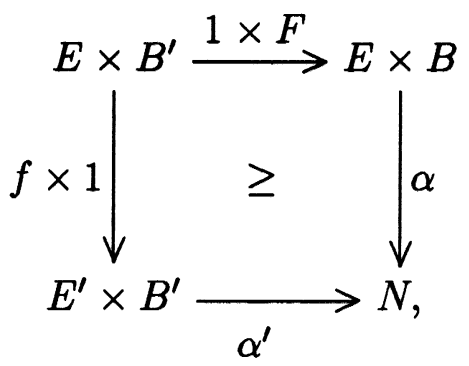

where $\leq$ is the partial order induced pointwise on $\mathbf{C}(A, N)$ by the partial order $\leq$ on $N$, and

- composition is composition in $\mathbf{C}$ for each component, thus $\langle g, G\rangle\langle f, F\rangle=$ $\langle g f, F G\rangle$,

\footnotetext{
${ }^{1}$ It is routine to generalise our construction by defining an order directly on the horn sets. For our purposes it suffices to consider concrete categories.
} 
define a category, which we shall denote $\mathbf{M}_{\mathbb{N}} \mathbf{C}$.

Proof: It is routine to verify that composition is well-defined, Let $\langle f, F\rangle:\langle E, B, \alpha\rangle \rightarrow\left\langle E^{\prime}, B^{\prime}, \alpha^{\prime}\right\rangle$ and $\langle g, G\rangle:\left\langle E^{\prime}, B^{\prime}, \alpha^{\prime}\right\rangle \rightarrow$ $\left\langle E^{\prime \prime}, B^{\prime \prime}, \alpha^{\prime \prime}\right\rangle$ be morphisms in $\mathbf{M}_{\mathbb{N}} \mathbf{C}$. Then by definition $\alpha^{\prime}(f \times$ 1) $\leq \alpha(1 \times F)$ and $\alpha^{\prime \prime}(g \times 1) \leq \alpha^{\prime}(1 \times G)$, we have $\alpha^{\prime \prime}(g f \times 1) \leq$ $\alpha(1 \times F G)$, and $\langle g f, F G\rangle$ is a morphism in $\mathbf{M}_{\mathbb{N}} \mathbf{C}$ from $\langle E, B, \alpha\rangle$ to $\left\langle E^{\prime \prime}, B^{\prime \prime}, \alpha^{\prime \prime}\right\rangle$. Identities and associativity are inherited from $\mathbf{C}$.

Since $\mathbf{C}$ is concrete, we interpret the ordering in the above diagram to mean that

$$
\text { for each } e \in E \text { and } b^{\prime} \in B^{\prime}, \quad \alpha^{\prime}\left\langle f e, b^{\prime}\right) \geq \alpha\left(e, F b^{\prime}\right\rangle \text {. }
$$

Our construction is a variant on that of Chu [Bar79], in which the above diagram commutes. Chu's construction is applied to any category with a symmetric monoidal closed structure, and a distinguished object $N$. We can extend our construction to a symmetric monoidal category C [DP91] rather than a category with finite products, and this enables us (see Section 6) to capture the notion of morphism between elementary Petri nets studied in [NRT90], and also to consider other mathematically interesting categories [HDP90].

Remark 4.2 Verity [Ver91] has proved that $\mathbf{M}_{\mathbb{N}} \mathbf{C}$ is enriched over $\mathbf{C}$.

Remark 4.3 It is evident that a categoy is also defined by the data:

- objects are the objects of $\mathbf{M}_{\mathbb{N}} \mathbf{C}$,

- a morphism from $\langle E, B, \alpha\rangle$ to $\left\langle E^{\prime}, B^{\prime}, \alpha^{\prime}\right\rangle$ is a pair $\left\langle f, F^{\prime}\right\rangle$ of morphisms in $\mathbf{C}$ such that $f: E \rightarrow E^{\prime}, F: B^{\prime} \rightarrow B$ and

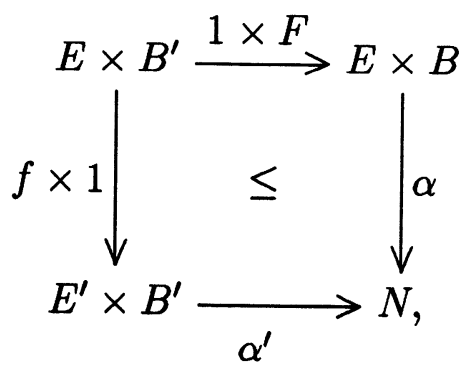


thus for each $e \in E$ and $b^{\prime} \in B^{\prime}$, we have $\alpha^{\prime}\left\langle f e, b^{\prime}\right) \leq \alpha\left(e, F b^{\prime}\right\rangle$,

- and composition given by composition in $\mathbf{C}$ for each component.

We shall denote this category $\mathbf{M}_{\mathbb{N}} \mathbf{C}^{*}$.

Proposition 4.4 $\mathrm{M}_{\mathbb{N}} \mathrm{C}$ is isomorphic to $\mathrm{M}_{\mathbb{N}} \mathrm{C}^{*}$.

Proof: There is an evident functor $\iota: \mathbf{M}_{\mathbb{N}} \mathbf{C} \rightarrow \mathbf{M}_{\mathbb{N}} \mathbf{C}^{*}$ taking the object $\langle E, B, \alpha\rangle$ to the object $\left\langle E, B, \alpha^{o p}\right\rangle^{2}$ and taking the morphism $\langle f, F\rangle$ to $\langle F, f\rangle$.

Thus

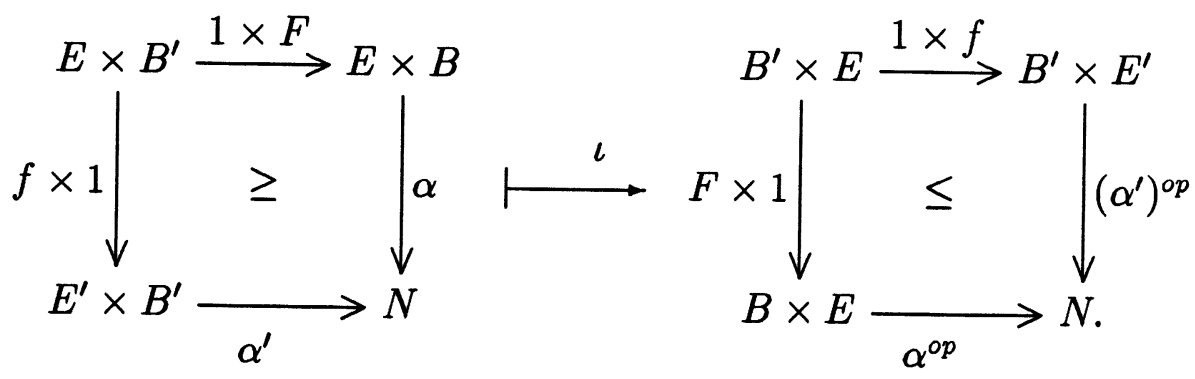

It is clear that $\iota$ is an isomorphism.

\section{$5 \quad \mathrm{M}_{\mathbb{N}} \mathrm{C}$ as a Model of Linear Logic}

If the category $\mathbf{C}$ and the object $N$ have appropriate structure, then we can define interesting structure on $\mathbf{M}_{\mathbb{N}} \mathbf{C}$. If $\mathbf{C}$ is Cartesian closed and has finite coproducts and $\langle N, \leq\rangle$ is a closed ordered monoid (that is, a partial order with a monotonic symmetric monoidal closed structure), then $\mathbf{M}_{\mathbb{N}} \mathbf{C}$ has finite products, finite coproducts and a symmetric monoidal closed structure. Note that these conditions are sufficient rather than necessary.

Part of the appeal of the category of elementary Petri nets NSet based on GC [BG90] lies in the fact that NSet is a sound model of linear logic in the sense of [DP89]. Given an interpretation of atomic formula as objects of NSet, we interpret the linear connective $\wedge$ by product, $\oplus$ by coproduct, $\otimes$ by the symmetric monoidal structure $\otimes$, linear implication $\multimap$ by the internal

\footnotetext{
${ }^{2}$ where $\alpha^{o p}\langle b, e\rangle=\alpha\langle e, b\rangle$ for any $e \in E$ and $b \in B$
} 
hom, par by a second symmetric monoidal structure $\square$ and linear negation by the functor $(-\multimap \perp)$, where $\perp$ is the unit of $\square$. Then whenever $\Gamma \vdash A$ in the fragment of linear logic comprising the structural rules, the rules for $\wedge, \oplus, \otimes, \multimap$ par and ! together with rules for intuitionistic negation, there is a morphism in NSet from the interpretation of $\Gamma$ to the interpretation of $A$.

The category of Petri nets which we introduce in this paper is constructed from $\mathbf{M}_{\mathbb{N}}$ Set, where Set is the category of sets and functions, and $\langle\mathbb{N}, \sqsubseteq\rangle$ is the set of natural numbers ordered by $\ldots 2 \sqsubseteq 1 \sqsubseteq 0$. Since Set is Cartesian closed and $\langle\mathbb{N}, \sqsubseteq\rangle$ is a closed ordered monoid (truncated subtraction being right adjoint to addition), $\mathbf{M}_{\mathbb{N}}$ Set has all finite products and coproducts, and is symmetric monoidal closed, affording a sound interpretation of intuitionistic linear logic.

\section{General Nets and Simulation Morphisms}

We now give a construction first sketched in [BG90]. We can regard a Petri net as an object $\left\langle\langle E, B\right.$, pre $\rangle,\left\langle E^{\prime}, B^{\prime}\right.$, post $\left.\rangle\right\rangle$ of $\mathbf{M}_{\mathbb{N}}$ Set $\times \mathbf{M}_{\mathbb{N}}$ Set for which $E=E^{\prime}$ and $B=B^{\prime}$. We can also regard it as an object of $\mathbf{M}_{\mathbb{N}}$ Set $\times$ $\mathbf{M}_{\mathbb{N}}$ Set $^{*}, \mathbf{M} v$ Set $^{*} \times \mathbf{M}_{\mathbb{N}}$ Set or $\mathbf{M}_{\mathbb{N}}$ Set $^{*} \times \mathbf{M}_{\mathbb{N}}$ Set ${ }^{*}$. Thus each of these four categories gives rise to a category with object set Petri. Accordingly, we have four related notions of morphism between Petri nets. In Section 7 we give an elegant characterisation of any of these categories as a limit in Cat, the category of small categories and functors. This implies that all relevant structure of $\mathbf{M}_{\mathbb{N}}$ Set lifts to each of our categories of nets.

In our earlier work we indicated how the morphisms in NSet, a category with object set the elementary Petri nets, expressed refinement or simulation. We also varied our morphisms, obtaining the categories NSet, NSet ${ }^{* 3}$, $\mathbf{N S e t}_{\perp} \leq$ and $\mathbf{N S e t}_{\leq} \gtreqless$. However, at that time it was unclear which notion of morphism was most appropriate.

In this paper we make precise the sense in which morphisms correspond to simulations, as Propositions 6.4 and 6.10 and Theorem 6.6 will demonstrate. A significant consequence of these results is that their proofs dictate the choices of the containments which we were previously unable to decide. Thus we shall work with the category with object set Petri which is a sub-

\footnotetext{
${ }^{3}$ called NSet ${ }^{c o}$ in $[$ Bro90]
} 
category of $\mathbf{M}_{\mathbb{N}}$ Set $\times \mathbf{M}_{\mathbb{N}}$ Set* $^{*}$, identifying the net $\langle E, B$, pre, post $\rangle$ with the object $\left\langle\langle E, B\right.$, pre $\rangle,\left\langle B, E\right.$, post $\left.\left.{ }^{o p}\right\rangle\right\rangle$. Note that we write $\leq$ for the usual ordering on $\mathbb{N}$.

Lemma 6.1 The following data:

- objects are general Petri nets, that is, elements of Petri,

- a morphism from $\langle E, B$, pre, post $\rangle$ to $\left\langle E^{\prime}, B^{\prime}, p r e^{\prime}, p o s t^{\prime}\right\rangle$ is a pair of functions $\langle f, F\rangle$ with $f: E \rightarrow E^{\prime}$ and $F: B^{\prime} \rightarrow B$ such that
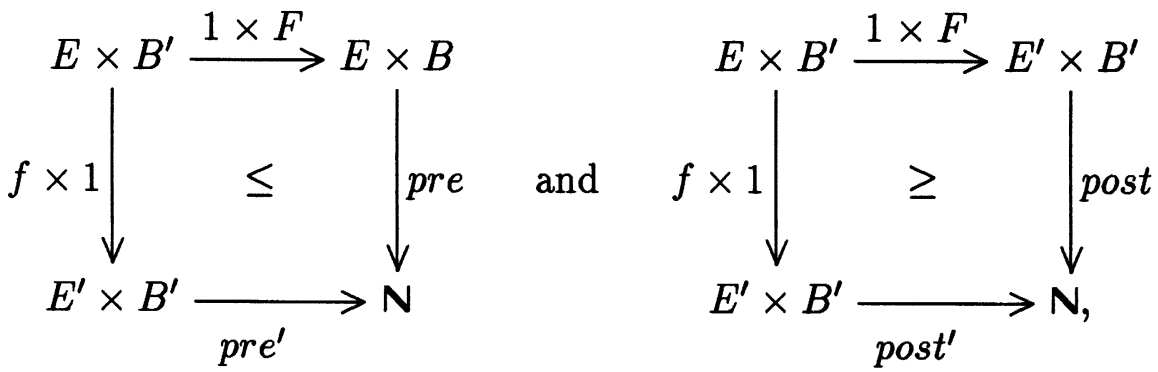

that is, for each $e \in E$ and each $b^{\prime} \in B^{\prime}$, we have

$$
\operatorname{pre}\left\langle e, F b^{\prime}\right\rangle \geq \operatorname{pre}^{\prime}\left\langle f e, b^{\prime}\right\rangle \text { and } \operatorname{post}\left\langle e, F b^{\prime}\right\rangle \leq \operatorname{post}^{\prime}\left\langle f e, b^{\prime}\right\rangle,
$$

- and composition is function composition in each component

define a category, which we shall denote GNet.

This result is a significant extension of that presented in [Bro90, BG90, $\mathrm{BG}$, since it allows us to treat general nets rather than the subclass of elementary nets.

In addition, we can exploit the generality of our framework by replacing Set by PSet, the category of sets and partial functions, obtaining a category PNet with object set Petri which is a full subcategory of $\mathbf{M}_{\mathbb{N}}$ PSet $\times$ $\mathbf{M}_{\mathbb{N}}$ PSet*. As the proof of Theorem 7.3 is independent of the base category $\mathbf{C}$, it is readily seen that $\mathbf{P N e t}$ is symmetric monoidal closed and has finite products and coproducts. The subcategory of PNet in which our inequalities are replaced by equality, $\mathbf{P N e t}^{=}$, has been studied in [NW91], and some relevant comparisons are made in [Bro90]. The full subcategory of $\mathbf{P N e t}^{=}$ with objects the elementary Petri nets is that studied in [NRT90]. Thus our 
framework, starting from linear logic and an abstract approach, encompasses several existing models.

We now prove the results which determined our choice of morphism in GNet. We prove them for events and sequences of events: their extension to multisets of events and sequences of multisets of events is straightforward. We first make some definitions which enable us to discuss simulation in a precise way.

Definition 6.2 Let $\langle N, M\rangle$ and $\left\langle N^{\prime}, M^{\prime}\right\rangle$ be marked Petri nets, and let $F$ be a function from $B^{\prime}$ to $B$. We say that the pair of markings $\left\langle M, M^{\prime}\right\rangle$ is $F$-ok if $M F \leq M^{\prime}$, that is, if we have

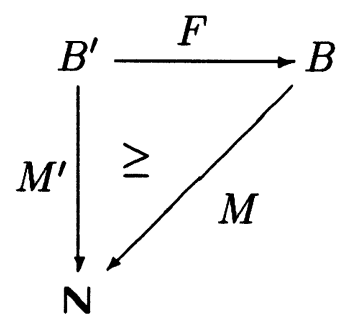

in Set

Definition 6.3 Let $\langle f, F\rangle: N \rightarrow N^{\prime}$ be a morphism in GNet, and for $i \in\{0, \ldots n+1\}$ let $\left\langle M_{i}, M_{i}^{\prime}\right\rangle$ be F-ok. Suppose that $M_{0} \stackrel{A_{0}}{\rightarrow} M_{1} \stackrel{A_{1}}{\sim} 1$ $M_{2} \ldots \stackrel{A_{n}}{\sim} M_{n+1}$ in $\mathrm{N}$.

The direct simulation of this evolution in $\mathrm{N}^{\prime}$ is $M_{0}^{\prime} \stackrel{f A_{0}}{\rightarrow}{ }_{1} M_{1}^{\prime} \stackrel{f A_{0}}{\rightarrow}{ }_{1} M_{2}^{\prime} \ldots \stackrel{f A_{n}}{\rightarrow} 1$ $M_{n+1}^{\prime}$.

A simulation of the above evolution in $\mathrm{N}^{\prime}$ is $M_{0}^{\prime} \stackrel{s_{0}}{\sim}{ }_{1} M_{1}^{\prime} \stackrel{s_{1}}{\rightarrow_{1}} M_{2}^{\prime} \ldots \stackrel{s_{n}}{\sim} M_{1} M_{n+1}^{\prime}$, where for $i \in\{0, \ldots, n\}, s_{i}$ is a finite sequence of transitions $t_{i_{j}}$ such that $\sum_{j} t_{i_{j}}=f A_{i}$.

$A$ weak simulation of the above evolution in $\mathrm{N}^{\prime}$ is $M_{0}^{\prime} \stackrel{v_{0}}{\sim} M_{1}^{\prime \prime} \stackrel{v_{1}}{\sim} M_{1}^{\prime \prime} \ldots \stackrel{v_{n}}{\sim} 1$ $M_{n+1}^{\prime \prime}$, where for $i \in\{0, \ldots n+1\},\left\langle M_{i}, M_{i}^{\prime \prime}\right\rangle$ is $F$-ok and $v_{i}$ is a finite sequence of transitions $w_{i_{j}}$ such that $f^{-1} \sum_{j} w_{i_{j}}=f A_{i}$.

Proposition 6.4 Let $\langle\mathrm{N}, M\rangle$ and $\left\langle\mathrm{N}^{\prime}, M^{\prime}\right\rangle$ be marked Petri nets and let $\langle f, F\rangle$ be a morphism from $\mathrm{N}$ to $\mathrm{N}^{\prime}$ in GNet such that $\left\langle M, M^{\prime}\right\rangle$ is $F$-ok. Then for all $e \in E, M \downarrow^{\mathrm{N}} e$ implies that $M^{\prime} \downarrow^{\mathrm{N}^{\prime}} f e$. 
Proof: Suppose that $M \downarrow^{\mathrm{N}} e$, that is $\forall b \in B .(M(b) \geq \operatorname{pre}\langle e, b\rangle)$. In particular, $\forall b^{\prime} \in B^{\prime}$ we have $M\left(F b^{\prime}\right) \geq \operatorname{pre}\left\langle e, F b^{\prime}\right\rangle$.

However, by the definition of $\langle f, F\rangle$,

$$
\forall e \in E, b^{\prime} \in B^{\prime} \text { we have pre }\left\langle e, F b^{\prime}\right\rangle \geq p r e^{\prime}\left\langle f e, b^{\prime}\right\rangle,
$$

and therefore $\forall b^{\prime} \in B^{\prime}$ we have $M\left(F b^{\prime}\right) \geq$ pre $\left\langle e, F b^{\prime}\right\rangle \geq p r e^{\prime}\left\langle f e, b^{\prime}\right\rangle$. Now, by hypothesis, $\left\langle M, M^{\prime}\right\rangle$ is $F$-ok, that is, $\forall b^{\prime} \in B^{\prime}$. $\left(M^{\prime}\left(b^{\prime}\right) \geq\right.$ $\left.M\left(F b^{\prime}\right)\right)$, hence $\forall b^{\prime} \in B^{\prime}$ we have $M^{\prime}\left(b^{\prime}\right) \geq M\left(F b^{\prime}\right) \geq$ pre $\left\langle e, F b^{\prime}\right\rangle \geq$ pre' $\left\langle f e, b^{\prime}\right\rangle$, which implies that $M^{\prime} \downarrow^{N^{\prime}} f e$.

If a pair of markings $\left\langle M, M^{\prime}\right\rangle$ is $F$-ok, then the net $\mathrm{N}^{\prime}$ with marking $M^{\prime}$ can simulate any one-step evolution of the net $\mathrm{N}$ with marking $M$, in the sense that whenever $\langle\mathrm{N}, M\rangle$ enables an event $e,\left\langle\mathrm{~N}^{\prime}, M^{\prime}\right\rangle$ enables the event $f e$. We now show that this holds for any sequence of events in $\mathrm{N}$, so that $\mathrm{N}^{\prime}$ can simulate any evolution of $\mathrm{N}$.

Definition 6.5 Let $\left\langle\mathrm{N}, M_{0}\right\rangle$ be a marked Petri net and let $e_{0}, e_{1}, \ldots, e_{n} \in E$. We say that $\left\langle\mathrm{N}, M_{0}\right\rangle$ enables the sequence $e_{0}, e_{1}, \ldots, e_{n}$, written $M_{0} \downarrow^{\mathrm{N}}$ $e_{0}, e_{1}, \ldots, e_{n}$, if there is a sequence $M_{0}, M_{1}, \ldots, M_{n+1}$ of markings of $\mathrm{N}$ such that:

$$
\forall i \in\{0, \ldots, n\} .\left(M_{i} \downarrow^{\mathrm{N}} e_{i} \text { and } M_{i} \stackrel{e_{i}}{\sim} M_{i+1}\right) .
$$

The following important result shows that $F$-ok-ness is preserved under evolution. A consequence of this result is that if $\langle\mathrm{N}, M\rangle$ enables a sequence of events $e_{0}, e_{1}, \ldots, e_{n}$ then $\left\langle\mathrm{N}^{\prime}, M^{\prime}\right\rangle$ enables the sequence $f e_{0}, f e_{1}, \ldots, f e_{n}$. Evidently for any marking $M$ of $\mathrm{N}$ we can construct a marking $M^{\prime}$ of $\mathrm{N}^{\prime}$ such that $\left\langle M, M^{\prime}\right\rangle$ is $F$-ok, and thus $\mathrm{N}^{\prime}$ can simulate any behaviour of $\mathrm{N}$.

Theorem 6.6 Let $\left\langle\mathrm{N}, M_{0}\right\rangle$ and $\left\langle\mathrm{N}^{\prime}, M_{0}^{\prime}\right\rangle$ be marked nets and let $\langle f, F\rangle$ be a morphism from $\mathrm{N}$ to $\mathrm{N}^{\prime}$ in GNet. If $\left\langle M_{0}, M_{0}^{\prime}\right\rangle$ is $F$-ok and $M_{0} \stackrel{e}{\sim}{ }_{1} M_{1}$, then $M_{0}^{\prime} \stackrel{f e}{\rightarrow} M_{1}^{\prime}$ and $\left\langle M_{1}, M_{1}^{\prime}\right\rangle$ is $F$-ok.

Proof: Suppose that $\left\langle M_{0}, M_{0}^{\prime}\right\rangle$ is $F$-ok and $M_{0} \stackrel{e}{\sim} M_{1} M_{1}$. Then $M_{0} \downarrow^{\mathrm{N}} e$ and therefore, by Proposition 6.4, $M_{0}^{\prime} \downarrow^{\mathbb{N}^{\prime}} f e$ and $M_{0}^{\prime} \stackrel{f e}{\sim} \gg_{1}$ $M_{1}^{\prime}$.

Now $M_{1}=M_{0}-\operatorname{pre}(e)+\operatorname{post}(e)$ and $M_{1}^{\prime}=M_{0}^{\prime}-\operatorname{pre}(f e)+$ post $^{\prime}(f e)$.

That is: 
$\forall b \in B .\left(M_{1}(b)=\left(M_{0}(b)-p r e\langle e, b\rangle\right)^{4}+p o s t\langle e, b\rangle\right) \quad$ and $\forall b^{\prime} \in B^{\prime} .\left(M_{1}^{\prime}\left(b^{\prime}\right)=\left(M_{0}^{\prime}\left(b^{\prime}\right)-p r e^{\prime}\left\langle f e, b^{\prime}\right\rangle\right)+\right.$ post $\left.^{\prime}\left\langle f e, b^{\prime}\right\rangle\right)$.

Now for each $b^{\prime} \in B^{\prime}$, we have

$$
\begin{aligned}
M_{1}^{\prime}\left(b^{\prime}\right) & =\left(M_{0}^{\prime}\left(b^{\prime}\right)-\operatorname{pre}^{\prime}\left\langle f e, b^{\prime}\right\rangle\right)+\operatorname{post}^{\prime}\left\langle f e, b^{\prime}\right\rangle \\
& \geq\left(M_{0}\left(F b^{\prime}\right)-\operatorname{pre}^{\prime}\left\langle f e, b^{\prime}\right\rangle\right)+\operatorname{post}^{\prime}\left\langle f e, b^{\prime}\right\rangle \text { as }\left\langle M_{0}, M_{0}^{\prime}\right\rangle \text { is } F-\text { ok } \\
& \geq\left(M_{0}\left(F b^{\prime}\right)-\operatorname{pre}^{\prime}\left\langle e, F b^{\prime}\right\rangle\right)+\operatorname{post}^{\prime}\left\langle f e, b^{\prime}\right\rangle \text { by definition of }\langle f, F\rangle \\
& \geq\left(M_{0}\left(F b^{\prime}\right)-\operatorname{pre}^{\prime}\left\langle e, F b^{\prime}\right\rangle\right)+\text { post }\left\langle e, F b^{\prime}\right\rangle \text { by definition of }\langle f, F\rangle \\
& =M_{1}\left(F b^{\prime}\right) .
\end{aligned}
$$

Thus $\forall b^{\prime} \in B^{\prime}$ we have $M_{1}^{\prime}\left(b^{\prime}\right) \geq M_{1}\left(F b^{\prime}\right)$, and so $\left\langle M_{1}, M_{1}^{\prime}\right\rangle$ is $F$-ok.

Corollary 6.7 Let $\left\langle\mathrm{N}, M_{0}\right\rangle$ and $\left\langle\mathrm{N}^{\prime}, M_{0}^{\prime}\right\rangle$ be marked Petri nets and let $\langle f, F\rangle$ be a morphism from $\mathrm{N}$ to $\mathrm{N}^{\prime}$ in GNet. If $\left\langle M_{0}, M_{0}^{\prime}\right\rangle$ is $F$-ok, then whenever $M_{0} \downarrow^{\mathrm{N}} e_{0}, e_{1}, \ldots, e_{n}$, we have $M_{0}^{\prime} \downarrow^{\mathrm{N}^{\prime}} f e_{0}, f e_{1}, \ldots, f e_{n}$.

Proof: By induction on the length of the sequence. For the inductive step, observe that if $M_{i} \downarrow^{\mathrm{N}} e_{i}$ and $M_{i} \stackrel{e_{i}}{\sim}{ }_{1} M_{i+1}$ and $\left\langle M_{i}, M_{i}^{\prime}\right\rangle$ is $F$-ok then $M_{i}^{\prime} \downarrow^{\mathrm{N}^{\prime}} f e_{i}$ (by Proposition 6.4), while $M_{i}^{\prime} \stackrel{f e_{i}}{\sim}{ }_{1} M_{i+1}^{\prime}$ and $\left\langle M_{i+1}, M_{i+1}^{\prime}\right\rangle$ is $F$-ok (by Theorem 6.6).

Thus if $\langle\mathrm{N}, M\rangle$ enables a sequence of events $e_{0}, e_{1}, \ldots, e_{n}$ then $\left\langle\mathrm{N}^{\prime}, M^{\prime}\right\rangle$ enables the sequence $f e_{0}, f e_{1}, \ldots, f e_{n}$. Evidently for any marking $M$ of $\mathrm{N}$ we can construct a marking $M^{\prime}$ of $\mathrm{N}^{\prime}$ such that $\left\langle M, M^{\prime}\right\rangle$ is $F$-ok, and thus $\mathrm{N}^{\prime}$ can simulate any behaviour of $\mathrm{N}$. Recall that a weak simulation of an evolution may include events which are not in the image of $f$ (that is, events which do not correspond directly to any event in the simulated net) but while it evolves under such events, $F$-ok-ness is preserved and so the simulating net $\mathrm{N}^{\prime}$ never loses the capacity to proceed with the direct simulation of the evolution of $\mathrm{N}$. Note that a direct simulation is a simulation, and that a simulation is a weak simulation. The concept of weak simulation allows us to consider simulations in which the simulated net $\mathrm{N}$ may idle (or stutter) while the simulating net proceeds with events which do not correspond directly to events of $\mathrm{N}$. In Section 6.1 we give an example to illustrate these concepts. The following proposition shows that we can extend the results above from direct simulations to weak simulations. The extension of Proposition 6.8 from events to 
transitions is straightforward.

Proposition 6.8 Let $\langle f, F\rangle: \mathrm{N} \rightarrow \mathrm{N}^{\prime}$ be a morphism in GNet. Let the pair $\left\langle M_{0}, M_{0}^{\prime}\right\rangle$ be $F$-ok. If $M_{0} \downarrow^{N} e_{0}, e_{1}, \ldots, e_{n}$ then $M_{0}^{\prime}$ enables any weak simulation of $e_{0}, e_{1}, \ldots, e_{n}$.

Proof: We show that whenever $M_{0} \stackrel{e}{\sim} 1 M_{1}$, there is a weak simulation $M_{0}^{\prime} \stackrel{*}{\sim} 1 M_{a}^{\prime} \stackrel{f e}{\sim}{ }_{1} M_{b}^{\prime} \stackrel{*}{\sim} 1 M_{c}^{\prime}$, where * stands for any transition of $\mathrm{N}^{\prime}$ which is disjoint from $f(E)$, and $\left\langle M_{1}, M_{c}^{\prime}\right\rangle$ is $F$ ok.

If $M_{0}^{\prime} \stackrel{*}{\rightarrow} 1 M_{a}^{\prime}$ then by definition of weak simulation, $\left\langle M_{0}, M_{a}^{\prime}\right\rangle$ is $F$-ok. By Proposition 6.4, $M_{a}^{\prime}$ enables $f e$.

If $M_{a}^{\prime} \stackrel{f e}{\sim}{ }_{1} M_{b}^{\prime}$ then, by Theorem 6.6, the pair $\left\langle M_{1}, M_{b}^{\prime}\right\rangle$ is $F$-ok. By definition of weak simulation, if $M_{b}^{\prime} \stackrel{*}{\sim} 1 M_{c}^{\prime}$ then $\left\langle M_{1}, M_{c}^{\prime}\right\rangle$ is $F$-ok.

We can repeat this argument for each of the transitions of the evolution $e_{0}, e_{1}, \ldots, e_{n}$, and the result follows.

Theorem 6.6 showed that whenever we have a morphism from $\mathrm{N}$ to $\mathrm{N}^{\prime}$ in GNet, $\mathrm{N}^{\prime}$ can simulate any evolution of $\mathrm{N}$. Ideally, we would like to have a converse to this result. That is, a result which states that if $\mathrm{N}^{\prime}$ can simulate any evolution of $\mathrm{N}$ then there is a morphism from $\mathrm{N}$ to $\mathrm{N}^{\prime}$ in GNet, since this would show that our morphisms exactly capture the independent notion of simulation. Unfortunately, as the following example shows, we cannot quite achieve this.

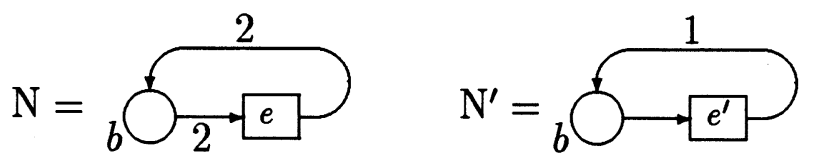

$\mathrm{N}^{\prime}$ can simulate any evolution of $\mathrm{N}$ but there is no morphism from $\mathrm{N}$ to $\mathrm{N}^{\prime}$ because post $(e, F b)=2>1=\operatorname{post}^{\prime}(f e, b)$ It transpires that the problem is that the first net has a condition, $b$, which is both a pre-condition and a post-condition of some event. If we restrict ourselves to the case where the first net does not have this property then we do indeed have a converse to Theorem 6.6 .

Definition 6.9 A net is loop free if for all events $e$, pre $(e) \cap \operatorname{post}(e)=\emptyset$. 
Proposition 6.10 Let $\mathrm{N}$ be a loop free net, let $\mathrm{N}^{\prime}$ be any net, let $f: E \rightarrow E^{\prime}$ and $F: B^{\prime} \rightarrow B$, and suppose that for all $F$-ok pairs of markings $\left\langle M, M^{\prime}\right\rangle$,

- $M \downarrow e$ implies that $M^{\prime} \downarrow f(e)$, and

- if $M \stackrel{e}{\sim} M_{1}$ and $M^{\prime} \stackrel{f(e)}{\sim} M_{1}^{\prime}$ then $\left\langle M_{1}, M_{1}^{\prime}\right\rangle$ is $F$-ok

then $\langle f, F\rangle$ is a morphism from $\mathrm{N}$ to $\mathrm{N}^{\prime}$ in GNet.

Proof: Let $M=\operatorname{pre}(e)$ and $M^{\prime}=M F$, so $\left\langle M, M^{\prime}\right\rangle$ is clearly $F$-ok. Then $M \downarrow^{\mathrm{N}} e$ whence, by assumption, $M^{\prime} \downarrow^{\mathrm{N}^{\prime}} f(e)$ and so for all $b^{\prime} \in B^{\prime}, \operatorname{pre}^{\prime}\left(f e, b^{\prime}\right) \leq M^{\prime}\left(b^{\prime}\right)=M\left(F b^{\prime}\right)=\operatorname{pre}\left(e, F b^{\prime}\right)$.

Now $M \stackrel{e}{\sim} \operatorname{post}(e)=M_{1}$ and $M^{\prime}=M F \stackrel{f(e)}{\sim}\left(M F-p r e^{\prime}(f e)\right)+$ $\operatorname{post}^{\prime}(f e)=M_{1}^{\prime}$ and by assumption $\left\langle M_{1}, M_{1}^{\prime}\right\rangle$ is $F$-ok. That is,

$M_{1}\left(F b^{\prime}\right)=\operatorname{post}\left(e, F b^{\prime}\right) \leq\left(\operatorname{pre}\left(e, F b^{\prime}\right)-p r e^{\prime}\left(f e, b^{\prime}\right)\right)+\operatorname{post}^{\prime}\left(f e, b^{\prime}\right)$.

If $\operatorname{post}\left(e, F b^{\prime}\right)=0$ then post $\left(e, F b^{\prime}\right) \leq \operatorname{post}^{\prime}\left(f e, b^{\prime}\right)$ and we are done. Otherwise, pre $\left(e, F b^{\prime}\right)=0$ by the assumption that $\mathrm{N}$ is loop free. However, we have shown that pre $\left(e, F b^{\prime}\right) \geq p r e^{\prime}\left(f e, b^{\prime}\right)$ and so $\operatorname{pre}^{\prime}\left(f e, b^{\prime}\right)=0$ as well whence,

$$
p o s t\left(e, F b^{\prime}\right) \leq\left(\operatorname{pre}\left(e, F b^{\prime}\right)-\operatorname{pre}^{\prime}\left(f e, b^{\prime}\right)\right)+\operatorname{post}^{\prime}\left(f e, b^{\prime}\right)=(0-0)+\operatorname{post}^{\prime}\left(f e, b^{\prime}\right)=\operatorname{post}^{\prime}\left(f e, b^{\prime}\right) .
$$

Thus $\langle f, F\rangle$ is a morphism from $\mathrm{N}$ to $\mathrm{N}^{\prime}$ in GNet.

The results of this section are important because they show that, not only do the marphisms of GNet have a meaningful computational interpretation, but also that [between loop-free nets] all simulations are captured by our morphisms. In other approaches [Win84, MM88b, NRT90], many simulations are not expressible as morphisms.

\subsection{A Practical Example of a Simulation}

We shall now exhibit a morphism from an unintelligent message handler to a more sophisticated message handler which can correct for the loss of a message in transit. This suffices to show that the message handler with 
error-correction can exhibit the simple behaviour of successively sending and delivering messages.

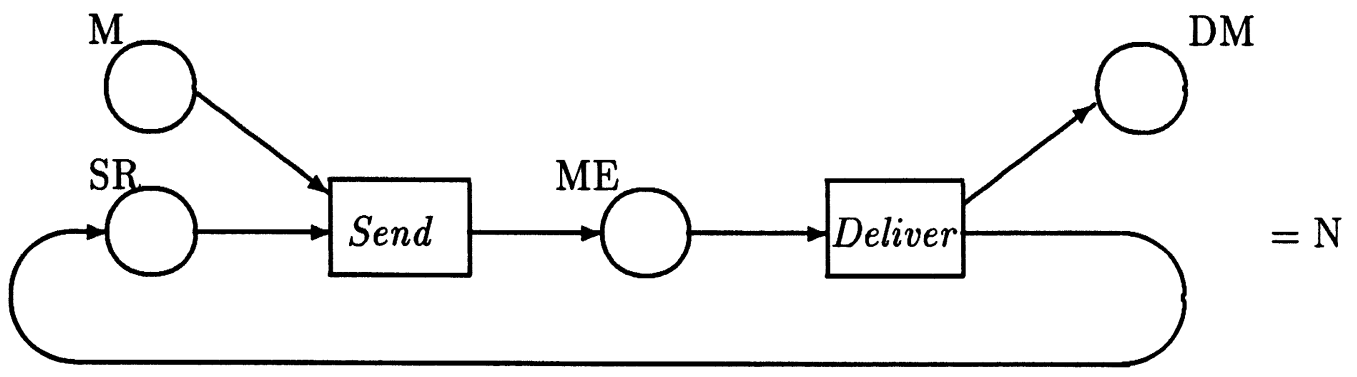

The simple message handler, with net $\mathrm{N}$, takes a message (M) and a flag that the sender is ready (SR), and dispatches the message to the ether via the event Send. The event Deliver accepts a message from the ether (ME) and delivers it (DM) to its destination, meanwhile setting the flag to indicate that the sender is once more ready to send a message. This net does not model any of the problems associated with a real message handler, a significant problem being the loss of messages from the ether.

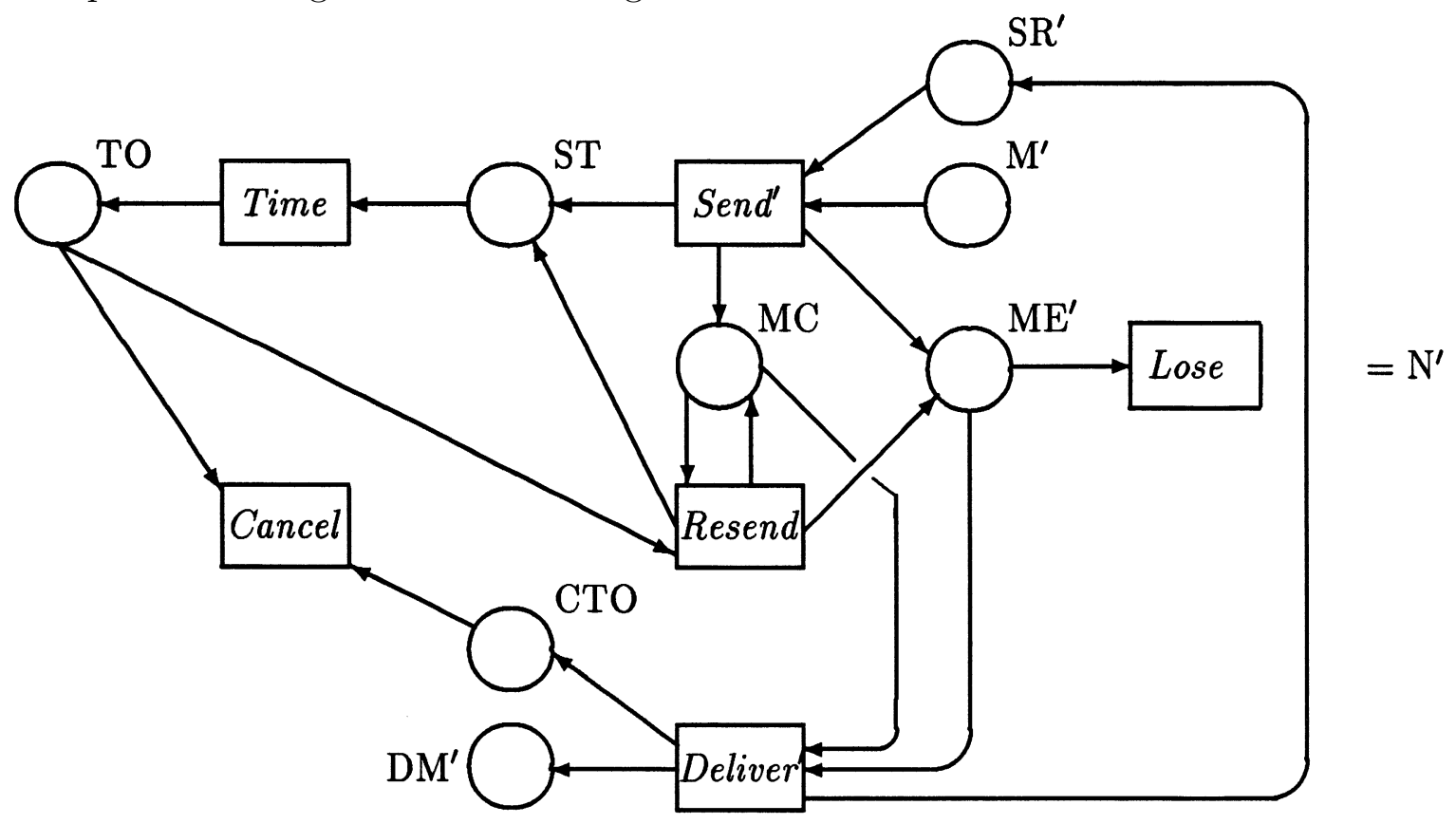

The error-correcting message handler resends a message which has been lost, and its net, $\mathrm{N}^{\prime}$, is more complex. The event Send' takes a message $\left(\mathrm{M}^{\prime}\right)$ and a flag that the sender is ready $\left(\mathrm{SR}^{\prime}\right)$, creates a copy of the message (MC) 
and dispatches the message to the ether $\left(\mathrm{ME}^{\prime}\right)$, meanwhile starting a timer (ST). The handler may Lose the message. Alternatively, the event Deliver may take a message from the ether, delete its copy, deliver it $\left(\mathrm{DM}^{\prime}\right)$, set a flag (CTO) cancelling the timeout signal and set the flag SR $\mathrm{SR}^{\prime}$ indicating that the sender is ready. The event Time is started by ST, and after a certain amount of time produces a timeout signal (TO). If a timeout signal is produced after the message has been delivered, CTO is set and the event Cancel forgets the timeout, resetting the flag CTO. If CTO is not marked when Time times out, then Cancel cannot occur but Resend becomes enabled. On receiving the timeout, Resend takes a message copy, recopies it and dispatches it to the ether, meanwhile restarting the timer.

Thus the message handler $\mathrm{N}^{\prime}$ can take a message and deliver it. If the message has not been delivered after a certain amount of time, it will resend the message. This enables it to correct the error of a message lost in the ether. It may also result in superfluous copies of a message being sent, since a timeout may occur when no message has been lost. To handle this error we would need a still more refined system, such as the alternating bit protocol [Mil89].

There is a morphism $\langle f, F\rangle$ in GNet from $\mathrm{N}$ to $\mathrm{N}^{\prime}$ given as follows:

$$
\begin{array}{ll}
f(\text { Send })=\text { Send }^{\prime} & f(\text { Deliver })=\text { Deliver }^{\prime} \\
F\left(\mathrm{SR}^{\prime}\right)=\mathrm{SR} & F\left(\mathrm{M}^{\prime}\right)=F(\mathrm{TO})=\mathrm{M} \\
F\left(M E^{\prime}\right)=F(\mathrm{MC})=F(\mathrm{ST})=\mathrm{ME} \quad \text { and } \quad & F(\mathrm{CTO})=F\left(\mathrm{DM}^{\prime}\right)=\mathrm{DM} .
\end{array}
$$

It is straightforward to check that the functions $f$ and $F$ defined above satisfy the required conditions, that is, for each event $e$ of $\mathrm{N}$ and each condition $b^{\prime}$ of $\mathrm{N}^{\prime}$, we have

$$
\operatorname{pre}\left\langle e, F b^{\prime}\right\rangle \geq \operatorname{pre}^{\prime}\left\langle f e, b^{\prime}\right\rangle \text { and } \operatorname{post}\left(e, F b^{\prime}\right) \leq \operatorname{post}^{\prime}\left\langle f e, b^{\prime}\right\rangle .
$$

The inequality is strict in two cases.

Theorem 6.6 indicates that for any pair $\left\langle M, M^{\prime}\right\rangle$ of $F$-ok markings, $\mathrm{N}^{\prime}$ with marking $M^{\prime}$ can simulate any behaviour of $\mathrm{N}$ with marking $M$. For example, $\mathrm{N}$ can evolve from marking $2 \mathrm{M}+\mathrm{SR}$ as follows:

$$
\begin{aligned}
& 2 \mathrm{M}+\mathrm{SR} \stackrel{\text { Send }}{\rightarrow 1} \mathrm{M}+\mathrm{ME} \stackrel{\text { Deliver }}{\rightarrow 1} \mathrm{M}+\mathrm{DM}+\mathrm{SR} \stackrel{\text { Send }}{\rightarrow 1} \mathrm{ME}+\mathrm{DM}+\mathrm{SR} \stackrel{\text { Deliver }}{\rightarrow} 2 \mathrm{DM} \\
& +\mathrm{SR} \text {. }
\end{aligned}
$$

The direct simulation of this evolution in $\mathrm{N}^{\prime}$ is 


$$
\begin{aligned}
& 2 \mathrm{M}^{\prime}+\mathrm{SR}^{\prime} \stackrel{\text { Send }^{\prime}}{\longrightarrow} \mathrm{M}^{\prime}+\mathrm{ME}^{\prime}+S T+M C \stackrel{\text { Deliver }}{\longrightarrow 1} \mathrm{M}^{\prime}+\mathrm{DM}^{\prime}+\mathrm{SR}^{\prime}+\mathrm{ST}+\mathrm{CTO} \\
& \stackrel{\text { Send }}{\rightarrow} \mathrm{ME}^{\prime}+\mathrm{DM}^{\prime}+\mathrm{MC}+2 \mathrm{ST}+\mathrm{CTO} \stackrel{\text { Deliver }}{\sim} 2 \mathrm{DM}^{\prime}+2 \mathrm{ST}+2 \mathrm{CTO} .
\end{aligned}
$$

It is easy to check that at each stage in this evolution, the corresponding pair of markings $\left\langle M, M^{\prime}\right\rangle$ is $F$-ok. The fact that the final marking of $\mathrm{N}^{\prime}$ includes the marking $2 \mathrm{ST}+2 \mathrm{CO}$ conflicts somewhat with our intuition. Because the events Time and Cancel are not in the image of $f$, they do not occur in the direct simulation. The evolution

$$
\begin{aligned}
2 \mathrm{M}^{\prime}+\mathrm{SR}^{\prime} \stackrel{\text { Send }}{\sim} \mathrm{M}^{\prime} & +\mathrm{ME}^{\prime}+S T+M C \stackrel{\text { Deliver }^{\prime}}{\sim 1} \mathrm{M}^{\prime}+\mathrm{DM}^{\prime}+\mathrm{SR}^{\prime}+\mathrm{ST}+\mathrm{CTO} \\
& \stackrel{\text { Time }}{\sim} \mathrm{M}^{\prime}+\mathrm{DM}^{\prime}+\mathrm{SR}^{\prime}+\mathrm{TO}+\mathrm{CTO} \stackrel{\text { Cancel }}{\sim} \mathrm{M}^{\prime}+\mathrm{DM}+\mathrm{SR}^{\prime} \\
& \stackrel{\text { Send }}{\sim} \mathrm{ME}^{\prime}+\mathrm{DM}^{\prime}+\mathrm{MC}+\mathrm{ST} \stackrel{\text { Deliver }}{\sim} 2 \mathrm{DM}^{\prime}+\mathrm{ST}+\mathrm{CTO} \\
& \stackrel{\text { Time }}{\sim} 2 \mathrm{DM}^{\prime}+\mathrm{SR}^{\prime}+\mathrm{TO}+\mathrm{COT} \stackrel{\text { Cancel }}{\sim} 2 \mathrm{DM}^{\prime}+\mathrm{SR}^{\prime}
\end{aligned}
$$

of $\mathrm{N}^{\prime}$ weakly simulates the evolution

$$
\begin{aligned}
& 2 \mathrm{M}+\mathrm{SR} \stackrel{\text { Send }}{\sim} \mathrm{M}+\mathrm{ME} \stackrel{\text { Deliver }}{\sim} \mathrm{M}+\mathrm{DM}+\mathrm{SR} \stackrel{\text { Send }}{\sim} \mathrm{ME}+\mathrm{DM}+\mathrm{SR} \stackrel{\text { Deliver }}{\sim} 2 \mathrm{DM} \\
& +\mathrm{SR} \text {. }
\end{aligned}
$$

Various other evolutions of $\mathrm{N}^{\prime}$ also evolve to marking $2 \mathrm{DM}^{\prime}+\mathrm{SR}^{\prime}$ while preserving $F$-ok-ness throughout.

The fact that there is a morphism in GNet from $\mathrm{N}$ to $\mathrm{N}^{\prime}$ proves that $\mathrm{N}^{\prime}$ correctly implements the behaviour specified by N. This motivates our choice of morphism in GNet.

\subsection{CCS Simulation}

We have described the morphisms in GNet as simulations on the grounds that a morphism between two nets $\mathrm{N}$ and $\mathrm{N}^{\prime}$ verifies that $\mathrm{N}^{\prime}$ can simulate any behaviour of $\mathrm{N}$. In fact, we can also understand our morphisms as simulations analogous to those of labelled transition systems such as CCS [Mil89]. We recall the definition of a strong bisimulation in CCS.

Definition 6.11 Let $\langle\mathcal{P}, \mathcal{A}\rangle$ be a labelled transition system. A binary relation $S \subseteq \mathcal{P} \times \mathcal{P}$ is a strong bisimulation if $(P, Q) \in S$ implies that, for all $\alpha \in \mathcal{A}$ : 
- whenever $P \stackrel{\alpha}{\longrightarrow} P^{\prime}$ then, for some $Q^{\prime}, Q \stackrel{\alpha}{\longrightarrow} Q^{\prime}$ and $\left(P^{\prime}, Q^{\prime}\right) \in S$ and

- whenever $Q \stackrel{\alpha}{\longrightarrow} Q^{\prime}$ then, for some $P^{\prime}, P \stackrel{\alpha}{\longrightarrow} P^{\prime}$ and $\left(P^{\prime}, Q^{\prime}\right) \in S$.

Similarly, one can define a simulation as follows.

Definition 6.12 Let $\langle\mathcal{P}, \mathcal{A}\rangle$ be a labelled transition system. A binary relation $S \subseteq \mathcal{P} \times \mathcal{P}$ is a simulation if $(P, Q) \in S$ implies that, for all $\alpha \in \mathcal{A}$ :

- whenever $P \stackrel{\alpha}{\longrightarrow} P^{\prime}$ then, for some $Q^{\prime}, Q \stackrel{\alpha}{\longrightarrow} Q^{\prime}$ and $\left(P^{\prime}, Q^{\prime}\right) \in S$.

In fact, we require a slight generalisation of this definition which extends simulation to a relation between states of different transition systems.

Definition 6.13 Let $\langle\mathcal{P}, \mathcal{A}\rangle$ and $\left\langle\mathcal{P}^{\prime}, \mathcal{A}^{\prime}\right\rangle$ be labelled transition systems and let $f$ be a function from $\mathcal{A}$ to $\mathcal{A}^{\prime}$. A binary relution $S \subseteq \mathcal{P} \times \mathcal{P}^{\prime}$ is a simulation relative to $f$ if $(P, Q) \in S$ implies that, for all $\alpha \in \mathcal{A}$ :

- whenever $P \stackrel{\alpha}{\longrightarrow} P^{\prime}$ then, for some $Q^{\prime} \in \mathcal{P}^{\prime}, Q \stackrel{f \alpha}{\longrightarrow} Q^{\prime}$ and $\left(P^{\prime}, Q^{\prime}\right) \in S$.

Now, it is well known that a Petri Net $\mathrm{N}$ may be viewed as a transition system $\langle\operatorname{Mark}(\mathrm{N}), E\rangle$ with transition relation given by $M_{0} \stackrel{e}{\longrightarrow} M_{1}$ if $M_{0} \downarrow^{N} e$ and $M_{1}=M_{0}-\operatorname{pre}(e)+\operatorname{post}(e)$. Observe that $F$-ok is a relation between $\operatorname{Mark}(\mathrm{N})$ and $\operatorname{Mark}\left(\mathrm{N}^{\prime}\right)$.

Proposition 6.14 Let $\langle f, F\rangle$ be a morphism from a net $\mathrm{N}$ to a net $\mathrm{N}^{\prime}$ in GNet. Then $F$-ok is a simulation relative to $f$ between $\langle\operatorname{Mark}(\mathrm{N}), E\rangle$ and $\left\langle\operatorname{Mark}\left(\mathrm{N}^{\prime}\right), E^{\prime}\right\rangle$.

Proof: Follows immediately from Proposition 6.4 and Theorem 6.6.

Moreover, we can generalise the definition of strong bisimulation in an analogous way:

Definition 6.15 Let $\langle\mathcal{P}, \mathcal{A}\rangle$ and $\left\langle\mathcal{P}^{\prime}, \mathcal{A}^{\prime}\right\rangle$ be labelled transition systems, let $f$ be a function from $\mathcal{A}$ to $\mathcal{A}^{\prime}$ and let $g$ be a function from $\mathcal{A}^{\prime}$ to $\mathcal{A}$. A binary relation $S \subseteq \mathcal{P} \times \mathcal{P}^{\prime}$ is a bisimulation relative to $(f, g)$ if $(P, Q) \in S$ implies that, for all $\alpha \in \mathcal{A}, \alpha^{\prime} \in \mathcal{A}^{\prime}$ : 
- whenever $P \stackrel{\alpha}{\longrightarrow} P^{\prime}$ then, for some $Q^{\prime} \in \mathcal{P}^{\prime}, Q \stackrel{f \alpha}{\longrightarrow} Q^{\prime}$ and $\left(P^{\prime}, Q^{\prime}\right) \in S$ and

- whenever $Q \stackrel{\alpha^{\prime}}{\longrightarrow} Q^{\prime}$ then, for some $P^{\prime} \in \mathcal{P}, Q \stackrel{g \alpha^{\prime}}{\longrightarrow} Q^{\prime}$ and $\left(P^{\prime}, Q^{\prime}\right) \in S$.

Proposition 6.16 Let $\mathrm{N}$ and $\mathrm{N}^{\prime}$ be nets, let $\langle f, F\rangle$ be a morphism from $\mathrm{N}$ to $\mathrm{N}^{\prime}$ in GNet and let $\langle g, G\rangle$ be a morphism from $\mathrm{N}^{\prime}$ to $\mathrm{N}$ in GNet. Then the relation $\sim \subseteq \operatorname{Mark}(\mathrm{N}) \times \operatorname{Mark}\left(\mathrm{N}^{\prime}\right)$ given by:

$$
M \sim M^{\prime} \text { if }\left\langle M, M^{\prime}\right\rangle \text { is } F \text {-ok and }\left\langle M^{\prime}, M\right\rangle \text { is } G \text {-ok }
$$

is a bisimulation relative to $(f, g)$.

\section{Structure in GNet}

The category $\mathbf{M}_{\mathbb{N}}$ Set, and the isomorphic category $\mathbf{M}_{\mathbb{N}}$ Set* have considerable categorical structure. In particular, they have finite products and coproducts and a symmetric monoidal closed structure [DP91]. An important issue is the extent to which this structure lifts to our category GNet. We now give an elegant characterisation of GNet as a limit in Cat, which leads to an easy proof that GNet has finite products, finite coproducts and a symmetric monoidal structure induced by those in $\mathbf{M}_{\mathbb{N}}$ Set. The proof closely follows the proof that NC inherits the structure of GC (see [Bro90, BG90, BG]).

Lemma 7.1 GNet is the pullback in Cat of the forgetful functor $\mathcal{U}: \mathbf{M}_{\mathbb{N}}$ Set + Set $\times$ Set $^{o p}$ along itself (a kernel pair).

Proof: First recall that $\mathbf{M}_{\mathbb{N}}$ Set is isomorphic to $\mathbf{M}_{\mathbb{N}}$ Set $^{*}$, and pullbacks are only defined up to isomorphism. Thus the pullback in Cat of $\mathcal{U}: \mathbf{M}_{\mathbb{N}}$ Set $\rightarrow$ Set $\times$ Set $^{o p}$ along $\mathcal{U}^{*}: \mathbf{M}_{\mathbb{N}}$ Set $^{*} \rightarrow$ Set $\times$ Set $^{o p}$ is isomorphic to the pullback:

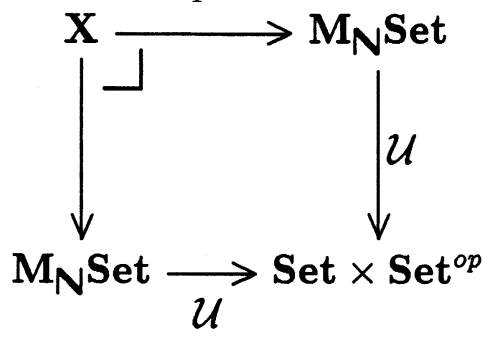


where $\mathcal{U}(\langle E, B, \alpha\rangle)=\langle E, B\rangle$ and $\mathcal{U}(\langle f, F\rangle)=\langle f, F\rangle$. Since Cat is Cartesian, this pullback is the equaliser of the arrows $\mathcal{U} \pi_{0}$ and $\mathcal{U} \pi_{1}$ from $\mathbf{M}_{\mathbb{N}}$ Set $\times \mathbf{M}_{\mathbb{N}}$ Set to Set $\times$ Set $^{o p}$. Hence

$O b(\mathbf{X})=\left\{\left\langle A_{0}, A_{1}\right\rangle \in O b\left(\mathbf{M}_{\mathbb{N}} \mathbf{S e t}\right) \times O b\left(\mathbf{M}_{\mathbb{N}} \mathbf{S e t}\right) \mid \mathcal{U} A_{0}=\mathcal{U} A_{1}\right\}$,

and similarly an arrow in $\mathbf{X}$ from $\left\langle A_{0}, A_{1}\right\rangle$ to $\left\langle A_{0}^{\prime}, A_{1}^{\prime}\right\rangle$ is a pair $\langle\langle f, F\rangle,\langle g, G\rangle\rangle$ with $\langle f, F\rangle: A_{0} \rightarrow A_{0}^{\prime}$ in $\mathbf{M}_{\mathbb{N}}$ Set and $\langle g, G\rangle$ : $A_{1} \rightarrow A_{1}^{\prime}$ in $\mathbf{M}_{\mathbb{N}}$ Set such that $\mathcal{U}\langle f, F\rangle=\mathcal{U}\langle g, G\rangle$, that is, such that $f=g$ and $F=G$.

Thus objects of $\mathbf{X}$ are of form $\left\langle\langle E, B, \alpha\rangle,\left\langle E, B, \alpha^{\prime}\right\rangle\right\rangle$ and a morphism in $\mathbf{X}$ from $\left\langle\left\langle E, B, \alpha_{0}\right\rangle,\left\langle E, B, \alpha_{1}\right\rangle\right\rangle$ to $\left\langle\left\langle E^{\prime}, B^{\prime}, \alpha_{0}^{\prime}\right\rangle,\left\langle E^{\prime}, B^{\prime}, \alpha_{1}^{\prime}\right\rangle\right\rangle$ is a pair $\langle\langle f, F\rangle,\langle f, F\rangle\rangle$ such that $\langle f, F\rangle:\left\langle E, B, \alpha_{0}\right\rangle \rightarrow\left\langle E^{\prime}, B^{\prime}, \alpha_{0}^{\prime}\right\rangle$ and $\langle f, F\rangle:\left\langle E, B, \alpha_{1}\right\rangle \rightarrow\left\langle E^{\prime}, B^{\prime}, \alpha_{1}^{\prime}\right\rangle$ in $\mathbf{M}_{\mathbb{N}}$ Set. Evidently, $\mathbf{X}$ is isomorphic to GNet.

Lemma $7.2 \mathcal{U}$ strictly preserves the product, coproduct and symmetric monoidal closed structure of $\mathbf{M}_{\mathbb{N}}$ Set.

Proof: Straightforward (compare the corresponding proof in [Bro90]).

Theorem 7.3 GNet has the products, coproducts and symmetric monoidal closed structure induced by those in $\mathbf{M}_{\mathbb{N}}$ Set.

Proof: The category of small categories with assigned finite products, assigned finite coproducts and strict monoidal closed structure, with morphisms the functors strictly preserving this structure, is monadic over Cat. The evident forgetful functor from it into Cat creates all limits, including kernel pairs. Since the product, coproduct and symmetric monoidal closed structure of $\mathbf{M}_{\mathbb{N}}$ Set are strictly preserved by $\mathcal{U}$ (Lemma 7.2), and since GNet is the kernel pair of $\mathcal{U}$ (Lemma 7.1), GNet has the products, coproducts and symmetric monoidal closed structure induced by those in $\mathbf{M}_{\mathbb{N}}$ Set.

This result has two consequences. Firstly, we maintain the connection with linear logic, since GNet is a sound model of the fragment of linear logic 
comprising the structural rules and the rules for $\wedge, \oplus, \otimes$ and $\multimap$. Secondly, we have obtained several constructors on general Petri nets. If $\mathrm{N}$ and $\mathrm{N}^{\prime}$ are nets, then their product $\mathrm{N} \times \mathrm{N}^{\prime}$, their coproduct $\mathrm{N}+\mathrm{N}^{\prime}$, their tensor product $\mathrm{N} \otimes \mathrm{N}^{\prime}$ and the implication $\mathrm{N} \multimap \mathrm{N}^{\prime}$ can all be interpreted as nets. To interpret the evolution of the compound net, we require an appropriate way of combining the markings of its component nets. In general, several possibilities exist, and decisions are hard to make using intuition alone. In Section 10 we resolve this issue using a category of marked nets.

\subsection{The Product of two Nets}

As we have seen, the product in GNet is induced by that in $\mathbf{M}_{\mathbb{N}}$ Set. Thus the product in GNet of nets $\left\langle E_{0}, B_{0}\right.$, pre $_{0}$, post $\left.t_{0}\right\rangle$ and $\left\langle E_{1}, B_{1}\right.$, pre $_{1}$, post $\left._{1}\right\rangle$ is $\left\langle E_{0} \times E_{1}, B_{0}+B_{1}\right.$,pre,post $\rangle$, where

$$
\begin{gathered}
\text { pre }\left\langle e_{0}, e_{1}\right\rangle=\sum_{b_{0} \in B_{0}} \operatorname{pre}_{0}\left\langle e_{0}, b_{0}\right\rangle\left(b_{0}, 0\right)+\sum_{b_{1} \in B_{1}} \operatorname{pre}_{1}\left\langle e_{1}, b_{1}\right\rangle\left(b_{1}, 1\right), \quad \text { and } \\
\text { post }\left\langle e_{0}, e_{1}\right\rangle=\sum_{b_{0} \in B_{0}} \operatorname{post}_{0}\left\langle e_{0}, b_{0}\right\rangle\left(b_{0}, 0\right)+\sum_{b_{1} \in B_{1}} \operatorname{post}_{1}\left\langle e_{1}, b_{1}\right\rangle\left(b_{1}, 1\right) .
\end{gathered}
$$

The precise details of the construction are given in Appendix B.

An event in the product net is the synchronisation of two events, one in each of the component nets: that is, a firing in the product net is the concurrent firing of an event in the first component and an event in the second. We relate the behaviour of the net $\mathrm{N}_{0} \times \mathrm{N}_{1}$ to that of its component nets neatly via Proposition 7.4, which shows that the behaviour of a product net is the product of the behaviours of its component nets.

Proposition 7.4 A marking $M$ of $\mathrm{N}_{0} \times \mathrm{N}_{1}$ can evolve by a multiset of events $A$ over $E$ to a marking $M^{\prime}$ if and only if for $i=0$ and $i=1$, the marking $i n_{i}^{o p} M$ can evolve in $\mathrm{N}_{i}$ by the multiset of events $\pi_{i} A$ to the marking $i n_{i}^{o p} M^{\prime}$.

Constructing products is essential for modelling parallel compositions. We give a simple example of the product of two nets. We give a more complex example of the use of the product in Section 8.

Example 7.5 Consider the two nets $\mathrm{N}_{0}$ and $\mathrm{N}_{1}$ given below: 

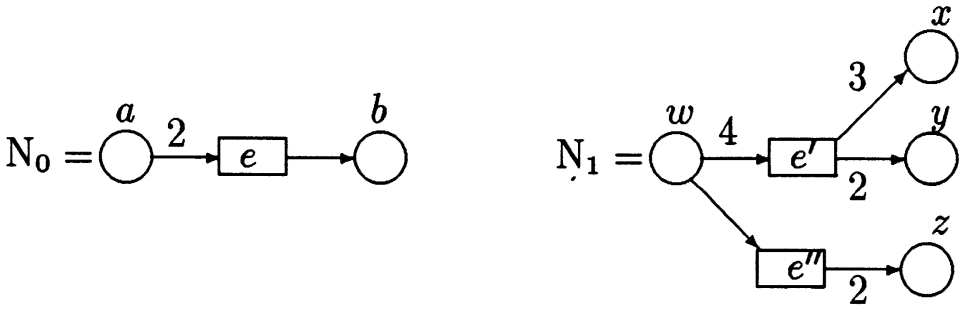

The product net $\mathrm{N}_{0} \times \mathrm{N}_{1}$ has event set $E=\left\{\left\langle e, e^{\prime}\right\rangle,\left\langle e, e^{\prime \prime}\right\rangle\right\}$ and condition set $B=\{(a, 0),(b, 0),(w, 1),(x, 1),(y, 1),(z, 1)\}$. The pre- and post-condition relations of $\mathrm{N}_{0} \times \mathrm{N}_{1}$ are given by:

$$
\begin{array}{ll}
\operatorname{pre}\left\langle e, e^{\prime}\right\rangle=2(a, 0)+4(w, 1), & \operatorname{post}\left\langle e, e^{\prime}\right\rangle=(b, 0)+3(x, 1)+2(y, 1), \\
\operatorname{pre}\left\langle e, e^{\prime \prime}\right\rangle=2(a, 0)+(w, 1), & \text { and } \quad \operatorname{post}\left\langle e, e^{\prime \prime}\right\rangle=(b, 0)+2(z, 1) .
\end{array}
$$

Thus the product net $\mathrm{N}_{0} \times \mathrm{N}_{1}$ is given by:

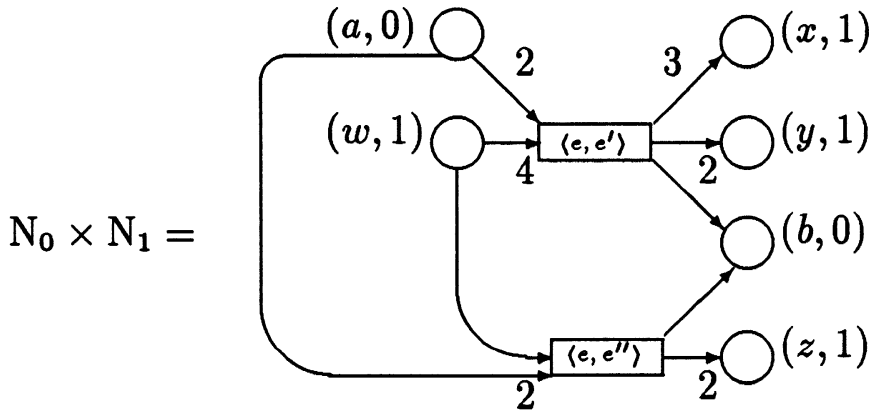

Remark 7.6 The product of two nets in GNet is their synchronous product [Win87].

\subsection{The coproduct of two nets}

The coproduct in GNet is also induced by that in $\mathbf{M}_{\mathbb{N}}$ Set. Thus the coproduct in GNet of nets $\left\langle E_{0}, B_{0}\right.$, pre $_{0}$, post $\left._{0}\right\rangle$ and $\left\langle E_{1}, B_{1}\right.$, pre $_{1}$, post $\left._{1}\right\rangle$ is $\left\langle E_{0}+E_{1}, B_{0} \times B_{1}\right.$, pre, post $\rangle$, where

$$
\begin{aligned}
& \operatorname{pre}\left(e_{0}, 0\right)=\sum_{b_{0} \in B_{0}} \sum_{b_{1} \in B_{1}} \text { pre }_{0}\left\langle e_{0}, b_{0}\right\rangle\left\langle b_{0}, b_{1}\right\rangle \text { and } \\
& \operatorname{post}\left(e_{0}, 0\right)=\sum_{b_{0} \in B_{0}} \sum_{b_{1} \in B_{1}} \operatorname{post}_{0}\left\langle e_{0}, b_{0}\right\rangle\left\langle b_{0}, b_{1}\right\rangle,
\end{aligned}
$$

and similarly for $\left(e_{1}, 1\right)$. In general the coproduct is not an appealing construct. However, we make considerable use of the special case $\mathrm{N}+\perp$, where 
$\perp=\langle 1,1,0, o\rangle$. Thus $\perp$ is the net consisting of one event $*$ and one condition $*$, with zero pre- and post-condition relations: it is the unit of $\otimes$, the symmetric monoidal structure induced in GNet by that in $\mathbf{M}_{\mathbb{N}}$ Set (described in Appendix A). We shall call the event with empty pre- and post-conditions the idling event, denoted $*$ or $\langle *, *\rangle$ according to context. Now we have

$$
\begin{aligned}
& \mathrm{N}+\perp=\left\langle E+\{*\}, B \times\{*\}, \text { pre }^{\prime}, \text { post }^{\prime}\right\rangle, \text { where } \operatorname{pre}^{\prime}\left(e_{0}, 0\right)= \\
& \sum_{b \in B} \operatorname{pre}(e, b)\langle b, *\rangle \cong \operatorname{pre}(e),
\end{aligned}
$$

and so on. For brevity, we shall write $\mathrm{N}_{\perp}$ for $\mathrm{N}+\perp$ and identify $E+\{*\}$ with $E \cup\{*\}$, and $B \times\{*\}$ with $B$.

\subsection{Expressing Synchrony and Asynchrony}

We have seen that an event $\left\langle e_{0}, e_{1}\right\rangle$ in the product net $\mathrm{N}_{0} \times \mathrm{N}_{1}$ is enabled whenever the events $e_{0}$ and $e_{1}$ in the component nets are both enabled at the same time. Thus events of the product net correspond to synchronisations of events in the component nets. Our next example demonstrates how events may occur asynchronously in a product net.

\section{Definition 7.7}

An event $\left\langle e_{0}, e_{1}\right\rangle$ of the product net $\mathrm{N}_{0} \times \mathrm{N}_{1}$ is asynchronous if for either $i=0$ or $i=1$,

$$
\operatorname{pre}\left\langle e_{0}, e_{1}\right\rangle=i n_{i}\left(\operatorname{pre}\left(e_{i}\right)\right) \text { and } \operatorname{post}\left\langle e_{0}, e_{1}\right\rangle=i n_{i}\left(\operatorname{post}\left(e_{i}\right)\right),
$$

where for $i=0,1$ we extend the function in $n_{i}$ to multisets over $B_{i}$ in the evident way.

Thus an asynchronous event is an event of a product net whose firing concerns only one of the component nets of the product. We shall now illustrate how asynchronous events arise when we apply our categorical constructions to nets.

Example 7.8 Let $\mathrm{N}_{0}$ and $\mathrm{N}_{1}$ be the nets:

$$
\mathrm{N}_{0}=\stackrel{a}{\bigcirc} \rightarrow e_{0} \stackrel{2}{\longrightarrow} \stackrel{b}{\bigcirc} \quad \mathrm{N}_{1}=\stackrel{x}{\bigcirc} \stackrel{2}{e_{1}} \longrightarrow \stackrel{y}{\bigcirc} .
$$


The product net $\left(\mathrm{N}_{0}+\perp\right) \times\left(\mathrm{N}_{1}+\perp\right)$ is given by:

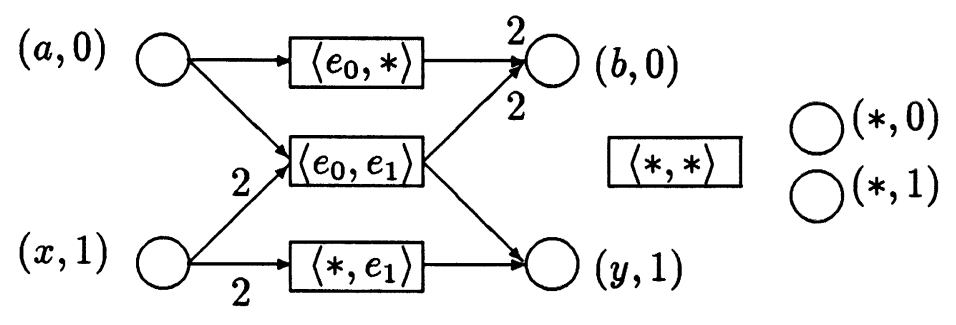

Observe that $\operatorname{pre}\left\langle e_{0}, *\right\rangle=i n_{0}\left(\operatorname{pre}(e)\right.$ and $\left.\operatorname{post}\left\langle e_{0}, *\right\rangle\right)=i n_{0}(\operatorname{post}(e))$. Thus $\left\langle e_{0}, *\right\rangle$, and similarly $\left\langle *, e_{1}\right\rangle$, are asynchronous events in the product net $\left(\mathrm{N}_{0}+\perp\right) \times\left(\mathrm{N}_{1}+\perp\right)$.

The event set of net $\left(\mathrm{N}_{0}+\perp\right) \times\left(\mathrm{N}_{1}+\perp\right)$ contains all possible synchronisations of events in $\mathrm{N}_{0}$ and $\mathrm{N}_{1}$, together with an asynchronous event corresponding to each event of either $\mathrm{N}_{0}$ or $\mathrm{N}_{1}$.

\section{A Compositional Theory for Petri Nets}

The categorical constructs we have defined can be used to give an algebraic means of building large nets from smaller components in such a way that the behaviour of the composite net can be expressed in terms of the behaviour of its components.

An important technique in building complex nets from simpler ones is that of restriction, by which we restrict a net to a specified subset of its possible behaviours. For example, if we limit our attention to a subset $E^{\prime}$ of the events of a net $\mathrm{N}=\langle E, B$,pre,post $\rangle$ then the net with this behaviour is $\mathrm{N}^{\prime}=\langle E, B, \operatorname{pre}(\iota), \operatorname{post}(\iota)\rangle$ where $\iota$ is the inclusion of $E^{\prime}$ in $E$, thus in Set:

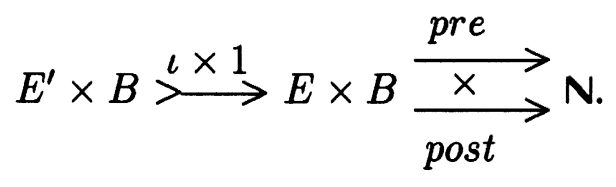

In particular, we often restrict ourselves to a subset of the events of a product net, thus restricting the possible synchronisations of its component nets. 
Thus if the event $\left\langle e_{0}, e_{1}\right\rangle \notin E^{\prime}$, the events $e_{0}$ and $e_{1}$ of the component nets may not synchronise. We specify such restrictions using a synchronisation function.

\section{Definition 8.1}

A synchronisation function on a product net $\mathrm{N}_{0} \times \mathrm{N}_{1}$ is a function $s$ : $E_{0} \times E_{1} \rightarrow\{0,1\}$

Definition 8.2 Let $s$ be a synchronisation function on the net $\mathrm{N}_{0} \times \mathrm{N}_{1}$. The restriction of $\mathrm{N}_{0} \times \mathrm{N}_{1}$ by s, written $\mathrm{N}_{0} \times \mathrm{N}_{1} \Gamma_{s}$, is the net with event set $E^{\prime}=\left\{\left\langle e_{0}, e_{1}\right\rangle \in E_{0} \times E_{1} \mid s\left\langle e_{0}, e_{1}\right\rangle=1\right\}$, condition set $B_{0}+B_{1}$ and pre-and post-condition relations given respectively by the restriction to $E^{\prime} \times\left(B_{0}+B_{1}\right)$ of the pre- and post-condition relations of $\mathrm{N}_{0} \times \mathrm{N}_{1}$.

Thus the net $\mathrm{N}_{0} \times \mathrm{N}_{1} \Gamma_{s}$ is such that

- $e_{0}$ and $e_{1}$ can synchronise if and only if $s\left\langle e_{0}, e_{1}\right\rangle=1$ and $e_{0}, e_{1} \neq *$,

- $e$ can occur asynchronously if and only if either $s(e, *)=1$ or $s(*, e)=$ 1 , and

- the idling event $\langle *, *\rangle$ is included if and only if $s\langle *, *\rangle=1$.

Remark 8.3 If $* \notin\left(E_{0} \cup E_{1}\right)$ then no events can occur asynchronously and we do not allow idling.

The following proposition gives a simple condition on synchronisation functions which ensures that simulation is preserved by restriction.

Proposition 8.4 Let $\langle f, F\rangle$ be a morphism in GNet from $\mathrm{N}_{0} \times \mathrm{N}_{1}$ to $\mathrm{N}_{0}^{\prime} \times \mathrm{N}_{1}^{\prime}$. Let $s$ and $s^{\prime}$ be synchronisation functions on $\mathrm{N}_{0} \times \mathrm{N}_{1}$ and $\mathrm{N}_{0}^{\prime} \times \mathrm{N}_{1}^{\prime}$ respectively. The restriction of $\langle f, F\rangle$ to $\mathrm{N}_{0} \times \mathrm{N}_{1}\left\lceil_{s}\right.$ factors through $\mathrm{N}_{0}^{\prime} \times \mathrm{N}_{1}^{\prime}\left\lceil_{s^{\prime}}\right.$ if and only if $s \leq s^{\prime} f$.

Proof: A morphism g making the diagram: 


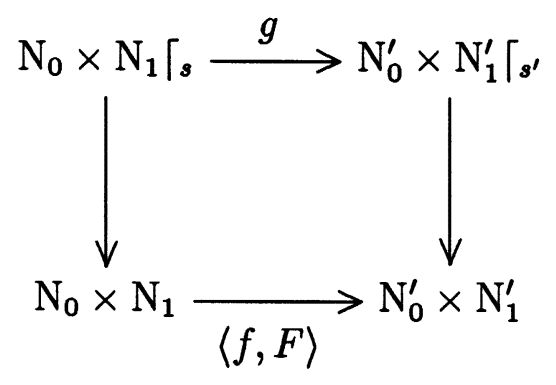

commute must be of form $\left\langle f^{\prime}, F\right\rangle$ where $f^{\prime}$ is the restriction of $f$ to the event set of $\mathrm{N}_{0} \times \mathrm{N}_{1} \Gamma_{s}$. The diagram clearly commutes if and only if whenever $\left\langle e_{0}, e_{1}\right\rangle$ is an event of $\mathrm{N}_{0} \times \mathrm{N}_{1}\left\lceil s\right.$, then $f^{\prime}\left\langle e_{0}, e_{1}\right\rangle$ is an event of $\mathrm{N}_{0}^{\prime} \times \mathrm{N}_{1}^{\prime}\left\lceil s^{\prime}\right.$, which is if and only if $s^{\prime} f^{\prime}\left\langle e_{0}, e_{1}\right\rangle \geq s\left\langle e_{0}, e_{1}\right\rangle$ for each event $\left\langle e_{0}, e_{1}\right\rangle$ of $\mathrm{N}_{0}^{\prime} \times \mathrm{N}_{1}^{\prime}\left\lceil s^{\prime}\right.$, which is if and only if $s^{\prime} f \geq s$.

Thus $\mathrm{N}_{0}^{\prime} \times \mathrm{N}_{1}^{\prime}\left\lceil_{s^{\prime}}\right.$ simulates $\mathrm{N}_{0} \times \mathrm{N}_{1}\left\lceil_{s}\right.$ if

- $\mathrm{N}_{0}^{\prime} \times \mathrm{N}_{1}^{\prime}$ simulates $\mathrm{N}_{0} \times \mathrm{N}_{1}$

- whenever $e_{0}$ and $e_{1}$ synchronise in $\mathrm{N}_{0} \times \mathrm{N}_{1} \Gamma_{s}$ then $\pi_{0} f\left\langle e_{0}, e_{1}\right\rangle$ and $\pi_{1} f\left\langle e_{0}, e_{1}\right\rangle$ synchronise in $\mathrm{N}_{0}^{\prime} \times \mathrm{N}_{1}^{\prime} \Gamma_{s^{\prime}}$ and

- whenever $\pi_{i} e$ is asynchronous in $\mathrm{N}_{0} \times \mathrm{N}_{1} \Gamma_{s}$ then $\pi_{i} f e$ is asynchronous in $\mathrm{N}_{1}^{\prime}\left\lceil s^{\prime}\right.$.

Note that the presence of the trivial event $\langle *, *\rangle$ is not preserved by morphisms in GNet.

Proposition 8.5 Let $s$ be a synchronisation function on $\mathrm{N}$ such that $\mathrm{N} \Gamma_{s}$ has event set $E^{\prime}$. Let $A$ be a multiset over $E^{\prime}$ and let $\widehat{A}$ be the multiset over $E$ such that $\widehat{A}(e)=A(e)$ for $e \in E^{\prime}$ and $\widehat{A}(e)=0$ otherwise. Let $C$ be a multiset over $E$, and $\bar{C}$ the restriction of $C$ to $E^{\prime}$. Then

- if $M \leadsto M^{\prime}$ in $\mathrm{N}\left\lceil_{s}\right.$ under $A$, then $M \leadsto M^{\prime}$ in $\mathrm{N}$ under $\widehat{A}$, and

- if $C(e)=0$ for all $e \in\left(E \backslash E^{\prime}\right)$ then whenever $M \sim^{C} M^{\prime}$ in $\mathrm{N}$, we have $M \leadsto \bar{C} M^{\prime}$.

Proof: Straightforward. 
Since every evolution of the restricted net is an evolution of the unrestricted net, restriction preserves safety properties (such as deadlock avoidance) but not liveness properties (such as reachability).

We illustrate the use of product, coproduct and restriction using an extended example related to the Jobshop example of [Mil89]p25. Our intention is to demonstrate the algebraic nature of our approach, rather than to make a direct analogy with CCS. We shall also illustrate the interaction of simulation morphisms with net constructors. It is an immediate consequence of the functoriality of the constructions $\times,+, \otimes$ and $\multimap$ that if for $i \in\{1, \ldots, n\}$ the net $\mathrm{N}_{i}^{\prime}$ simulates $\mathrm{N}_{i}$, then op $\left(\mathrm{N}_{1}^{\prime}, \ldots, \mathrm{N}_{n}^{\prime}\right)$ simulates op $\left(\mathrm{N}_{1}, \ldots, \mathrm{N}_{n}\right)$ where $o p$ may be any of $\wedge, \oplus, \otimes$ and $\multimap$. The interaction of simulation morphisms with restriction is given by Proposition 8.4. We shall specify a net representing a Jobshop by composing several smaller component nets. We first specify the behaviour of a mallet $(\mathrm{M})$ (drawing the condition $\mathrm{M}$ twice):

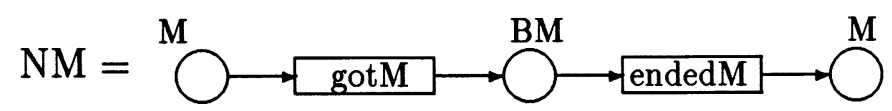

Thus, if a mallet is "got", it becomes a busy mallet (BM), and when its use is ended, it returns to its original state. It is readily proved (if not obvious) that the number of tokens on the net NM remains constant through out any behaviour of the net. It is the number of mallets in the jobshop. The specification of a hammer $(\mathrm{H})$ is similar:

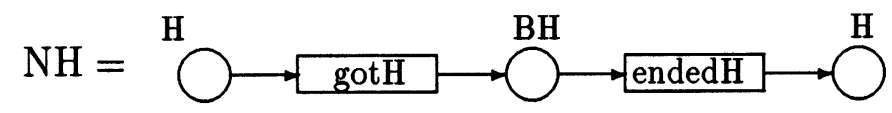

The behaviour of mallets and hammers together, or alone, might be described by the following net:

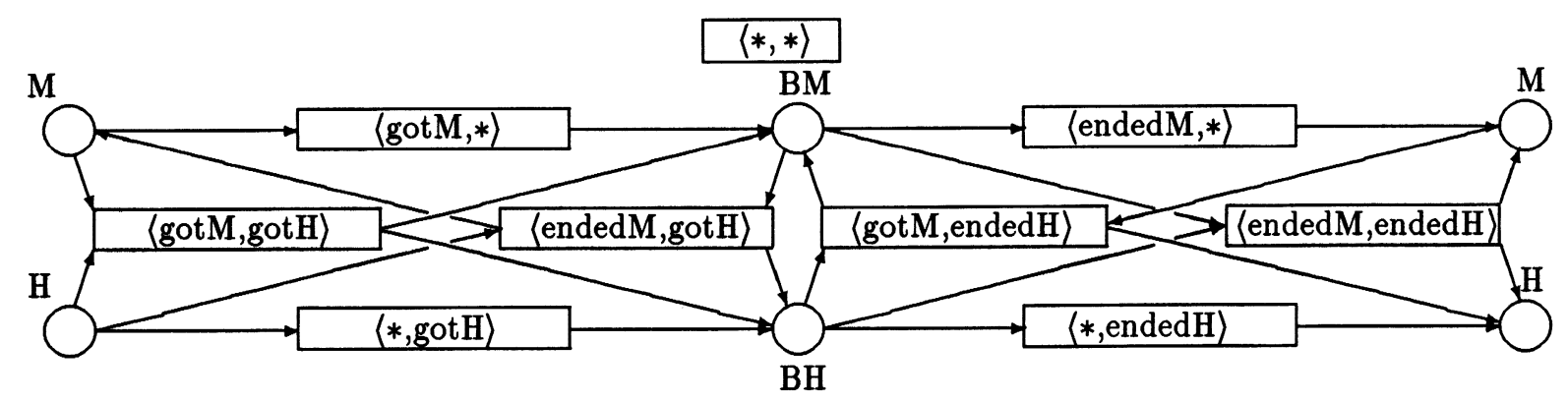

This net is $(\mathrm{NM}+\perp) \times(\mathrm{NH}+\perp)$, which we shall denote $\mathrm{NM}_{\perp} \times \mathrm{NH}_{\perp}$. In fact, we do not wish to force the synchronised use of mallets and hammers. 
To do so would imply either that each was used for the same length of time and never alone, or else that one was unnecessarily barred from use until the other became free. Similarly, there is no reason to synchronise the getting of a hammer with ending the use of a mallet. Therefore we shall restrict on the synchronised events, to give a net representing the asynchronous use of hammers and mallets.

We achieve this using the synchronisation function $s$ given by

$$
\begin{aligned}
& s\langle\operatorname{got} \mathrm{M}, *\rangle=s(*, \operatorname{got} \mathrm{H}\rangle=s\langle\text { endedM }, *\rangle=s\langle *, \text { ended } \mathrm{H}\rangle=1 \text { and } \\
& s\left\langle e_{0}, e_{1}\right\rangle=0 \text { otherwise. }
\end{aligned}
$$

Then the restricted net $\left(\mathrm{NM}_{\perp} \times \mathrm{NH}_{\perp}\right) \Gamma_{s}$ is such that

- no two events can synchronise

- every event of NM and NH can occur asynchronously, and

- the trivial event is not included.

The construction $\left(\mathrm{NM}_{\perp} \times \mathrm{NH}_{\perp}\right) \Gamma_{s}$ is comparable with the CCS parallel operator |. In CCS we restrict parallel compositions by requiring certain actions to occur only in synchronisation with specified complementary actions from separate processes. Here, we restrict to specified synchronisations of events from each component process and to specified asynchronous events.

We next specify a jobber $(\mathrm{J})$ in terms of the use of tools:

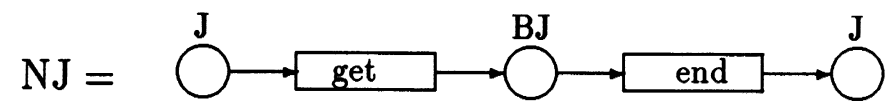

The jobber gets a tool, becoming busy (BJ). After using the tool, the jobber resumes ner initial state. The total number of tokens on this net is constant throughout any behaviour, and is the number of jobbers in our jobshop.

Consider the product net $\mathrm{NJ} \times\left(\mathrm{NM}_{\perp} \times \mathrm{NH}_{\perp} \Gamma_{s}\right)$. It has events:

$$
\begin{array}{llll}
\langle\text { get, gotM }, *\rangle & \langle\text { get, } * \text {, got } \mathrm{H}\rangle & \langle\text { end, endedM, } *\rangle & \langle\text { end, } * \text {, endedH }\rangle \\
\langle\text { get, endedM, } *\rangle & \langle\text { get, } * \text {, endedH }\rangle & \langle\text { end, gotM, } *\rangle & \langle\text { end, } * \text {, gotH }\rangle
\end{array}
$$

We want only the first four of these events, imposing the reasonable requirement that a jobber cannot get a tool unless a tool is simultaneously got, and 
cannot end the use of a tool unless the use of a tool is ended. Restricting to these four events, using the appropriate synchronisation function s', we obtain the net:

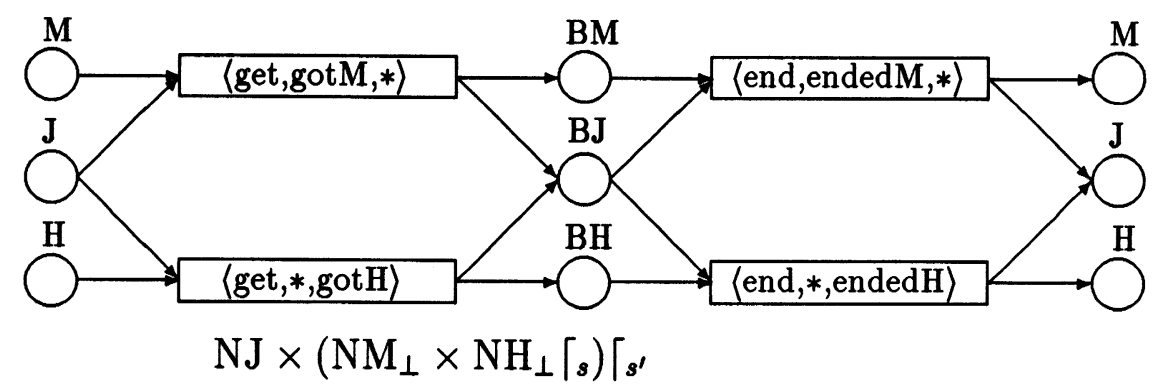

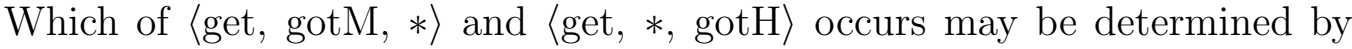
the environment, since either $\mathrm{H}$ or $\mathrm{M}$ may be marked. If both are marked, a non-deterministic choice is made internally by the system.

We can describe further interaction with the outside world, as Milner does, by considering how the jobber receives component pieces $(\mathrm{P})$ and outputs completed jobs (A), by specifying a new jobber thus:



Observe that this net can be thought of as a restriction of the product of NJ with a net specifying the behaviour of pieces. If we form the product $\mathrm{NJ}_{\perp}^{\prime} \times$ $\left(\mathrm{NM}_{\perp} \times \mathrm{NH}_{\perp}\left\lceil_{s}\right)\right.$ and restrict appropriately (by a synchronisation function we shall denote $t$ ), we obtain a net describing a jobber's behaviour with respect to hammers and mallets as before, but including also the interaction between the jobber and the pieces and completed jobs.

Now consider the following net, which assumes that pieces may constitute an easy job (EP) or a hard job (HP):

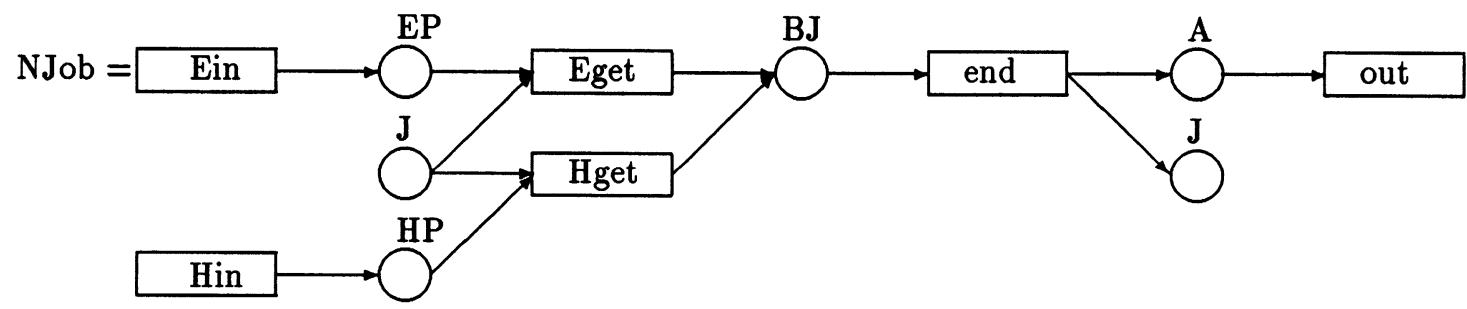


Now, NJob simulates $\mathrm{NJ}^{\prime}$, since there is a morphism $\langle f, F\rangle$ in GNet from NJob to $\mathrm{NJ}^{\prime}$ given by

$$
\begin{array}{llll}
f(\text { get })=\text { Eget } & f(\text { end })=\text { end } & \\
F(\mathrm{EP})=F(\mathrm{HP})=P & F(\mathrm{~J})=\mathrm{J} & F(\mathrm{BJ})=\mathrm{BJ} & F(\mathrm{~A})=\mathrm{A} .
\end{array}
$$

We shall assume that the jobber must use a mallet to assemble the hard pieces, but that either a hammer or a mallet may be used to assemble the easy pieces. With this requirement in mind, we restrict the product net $\mathrm{NJob} \times\left(\mathrm{NM}_{\perp} \times \mathrm{NH}_{\perp} \Gamma_{s}\right)$ by a synchronisation function we shall denote $t^{\prime}$, to obtain the net with events:

$$
\begin{array}{llll}
\langle\text { Eget, } * \text {, gotH }\rangle & \langle\text { Eget, gotH, } *\rangle & \langle\text { Hget, gotM, } *\rangle & \langle\text { end, endedH, } *\rangle \\
\langle\text { end, } * \text {, endedH }\rangle & \langle\text { Ein, } *, *\rangle & \langle\text { Hin }, *, *\rangle & \langle\text { out }, *, *\rangle
\end{array}
$$

and pre- and post-condition relations given by:

$$
\begin{aligned}
& \text { pre }\langle\text { Eget }, *, \operatorname{gotH}\rangle=\mathrm{EP}+\mathrm{H}+\mathrm{J} \quad \text { post }\langle\text { Eget }, *, \operatorname{gotH}\rangle=\mathrm{BJ}+\mathrm{BH} \quad \text { pre }\langle\text { Ein }, *, *\rangle=\emptyset \\
& \text { pre }\langle\text { Eget,gotM }, *\rangle=\mathrm{EP}+\mathrm{M}+\mathrm{J} \quad \text { post }\langle\text { Eget,gotM }, *\rangle=\mathrm{BJ}+\mathrm{BM} \quad \text { post }\langle\text { Ein }, *, *\rangle=\mathrm{EP} \\
& \text { pre }\langle\text { Hget,gotM }, *\rangle=\mathrm{HP}+\mathrm{M}+\mathrm{J} \quad \text { post }\langle\text { Hget,gotM }, *\rangle=\mathrm{BJ}+\mathrm{BM} \quad \text { pre }\langle\text { Hin }, *, *\rangle=\emptyset \\
& \text { pre }\langle\text { end,endedM }, *\rangle=\mathrm{BJ}+\mathrm{BM} \quad \operatorname{post}\langle\text { end,endedM }, *\rangle=\mathrm{M}+\mathrm{J}+\mathrm{A} \quad \operatorname{post}\langle\mathrm{Hin}, *, *\rangle=\mathrm{HP} \\
& \text { pre }\langle\text { end }, *, \mathrm{ended} \mathrm{H}\rangle=\mathrm{BJ}+\mathrm{BM} \quad \operatorname{post}\langle\mathrm{end}, *, \mathrm{ended} \mathrm{H}\rangle=\mathrm{H}+\mathrm{J}+\mathrm{A} \quad \operatorname{pre}\langle\text { out }, *, *\rangle=\mathrm{A}
\end{aligned}
$$

Now we have a morphism $\langle f, F\rangle \times 1$ in GNet from $\mathrm{NJ}^{\prime} \times\left(\mathrm{NM}_{\perp} \times \mathrm{NH}_{\perp} \Gamma_{s}\right)$ to $\mathrm{NJob} \times\left(\mathrm{NM}_{\perp} \times \mathrm{NH}_{\perp}\left\lceil_{s}\right)\right.$. Further, $t$ and $t^{\prime}$ satisfy the conditions of Lemma 8.4, and so $\langle f, F\rangle \times 1$ restricts to a morphism from $\mathrm{NJ}^{\prime} \times\left(\mathrm{NM}_{\perp} \times \mathrm{NH}_{\perp} \Gamma_{s}\right) \Gamma_{t}$ to $\mathrm{NJob} \times\left(\mathrm{NM}_{\perp} \times \mathrm{NH}_{\perp}\left\lceil_{s}\right)\left\lceil_{t^{\prime}}\right.\right.$. Thus we have a simulation of the compound net $\mathrm{NJ}^{\prime} \times\left(\mathrm{NM}_{\perp} \times \mathrm{NH}_{\perp} \Gamma_{s}\right) \Gamma_{t}$ by a compound net which has been extended to consider easy and hard jobs.

\subsection{The exponential of a net}

In [DP91] de P aiva modelled the linear modality "of course" by constructing a comonad! with the properties that,

$$
! A \otimes ! B=!(A \times B) \text { and } ! 1=\mathrm{I}
$$


By Theorem 7.3, this comonad lifts to a comonad! in GNet with the same properties. It has the following definition.

Definition 8.6 The exponential !N of the net $\mathrm{N}$ is the net $\left\langle E,\left(B^{*}\right)^{E}\right.$, pre $_{!}$, post $\left._{!}\right\rangle$ where $B^{*}$ is the free commutative monoid ove $B$, and if $\phi: E \rightarrow B^{*}$ is the function given by $\phi(e)=\sum_{B} m_{i} b_{i}$ then

$$
\operatorname{pre}_{!}(e, \phi)=\sum_{B} m_{i} \operatorname{pre}\left(e, b_{i}\right) \text { and } \operatorname{post}_{!}(e, \phi)=\sum_{B} m_{i} \operatorname{post}\left(e, b_{i}\right) \text {. }
$$

The net ! $\mathrm{N}$ has the same event set as $\mathrm{N}$ but a considerably larger condition set. We may understand the net $\mathrm{I}$ as a net in which the conditions have been "unwound" into all their possible requirements. This may be seen in the following example.

\section{Example 8.7}
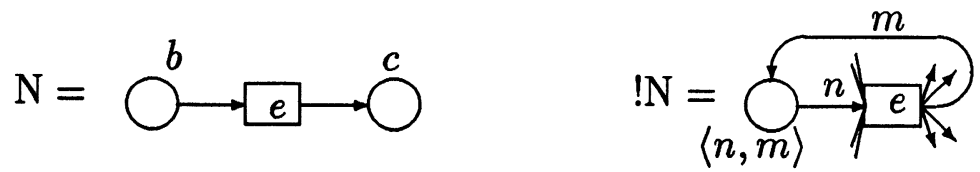

The net !N has one event $e$ and countably many conditions, labelled by pairs (because $B$ has two elements) of integers. The condition $\langle n, m\rangle$ appears in pre $(e)$ with multiplicity $n$ and in post $(e)$ with multiplicity $m$.

\subsection{The symmetric monoidal closed structure of GNet}

GNet has a symmetric monoidal closed structure induced by that in $\mathbf{M}_{\mathbb{N}}$ Set. The tensor product $\otimes$ and its right adjoint, linear implication $(\multimap)$, have the following definitions.

Definition 8.8 The tensor product $\left(N_{0} \otimes N_{1}\right)$ of two nets is the net $\left\langle E_{0} \times\right.$ $E_{1}, B_{0}^{E_{1}} \times B_{1}^{E_{0}}$, pre $_{0} \otimes$ pre $_{1}$, post $_{0} \otimes$ post $\left._{1}\right\rangle$ where,

$$
\begin{gathered}
\text { pre }_{0} \otimes \text { pre }_{1}\left\langle e_{0}, e_{1}, f, g\right\rangle=\text { pre }_{0}\left\langle e_{0}, f e_{1}\right\rangle+\text { pre }_{1}\left\langle e_{1}, g e_{0}\right\rangle \\
\text { post }_{0} \otimes \text { post }_{1}\left\langle e_{0}, e_{1}, f, g\right\rangle=\text { post }_{0}\left\langle e_{0}, f e_{1}\right\rangle+\text { post }_{1}\left\langle e_{1}, g e_{0}\right\rangle
\end{gathered}
$$


Its unit is the net $\mathrm{I}=\langle\{*\},\{*\}, 0,0\rangle$.

Definition 8.9 The linear implication $\left(\mathrm{N}_{0} \multimap \mathrm{N}_{1}\right)$ of two nets is the net $\left\langle E_{1}^{E_{0}} \times B_{0}^{B_{1}}, E_{0} \times B_{1}\right.$, pre $_{0} \multimap$ pre $_{1}$, post $_{0} \multimap$ post $\left._{1}\right\rangle$ where,

$$
\begin{gathered}
\left(\text { pre }_{0} \multimap \text { pre }_{1}\right)\left\langle f, F, e_{0}, b_{1}\right\rangle=\text { pre }_{0}\left\langle f e_{0}, b_{1}\right\rangle \ominus \text { pre }_{1}\left\langle e_{0}, F b_{1}\right\rangle \\
\left(\text { post }_{0} \multimap \text { post }_{1}\right)\left\langle f, F, e_{0}, b_{1}\right\rangle=\text { post }_{0}\left\langle f e_{0}, b_{1}\right\rangle \ominus \text { post }_{1}\left\langle e_{0}, F b_{1}\right\rangle
\end{gathered}
$$

and $\ominus$ is the truncated subtraction described in Appendix A.2.

\section{A Proof System for Nets}

In Section 8 we made implicit use of a language for nets. In this section, we shall describe how to extend this to a proof system for nets, based on Girard's intuitionistic linear logic [Gir87]. We first define a language $\mathcal{N}$ for nets by the following BNF,

$$
\mathcal{N}::=0|1| \mathrm{I}\left|\mathrm{N}_{i}\right| \mathrm{N} \oplus \mathrm{N}^{\prime}\left|\mathrm{N} \wedge \mathrm{N}^{\prime}\right| \mathrm{N} \otimes \mathrm{N}^{\prime}\left|\mathrm{N} \multimap \mathrm{N}^{\prime}\right| ! \mathrm{N} \mid \mathrm{N}\left\lceil_{s}\right.
$$

where the $\mathrm{N}_{i}$ and $s$ are chosen from two disjoint collections of constant symbols.

We shall interpet this language in GNet. The interpretation is parametric in a function $\tau$ which assigns an object of GNet, that is a Petri net, to each of the constant symbols $\mathrm{N}_{i}$ and a synchronisation function to each synchronisation symbol $s$. Each term of the language $\mathcal{N}$ is then interpreted by an object of GNet in the following way.

- $\llbracket \mathrm{N}_{i} \rrbracket=\tau\left(\mathrm{N}_{i}\right)$ for each constant symbol $\mathrm{N}_{i}$,

- $\llbracket \mathbf{1} \rrbracket=\mathbf{1}=\langle\{*\}, \phi, \phi, \phi\rangle$,

- $\llbracket \mathbf{0} \rrbracket=\mathbf{0}=\langle\phi,\{*\}, \phi, \phi\rangle$,

- $\llbracket \mathrm{I} \rrbracket=\mathrm{I}=\langle\{*\},\{*\}, 0,0\rangle$,

- $\llbracket \mathrm{N} \wedge \mathrm{N}^{\prime} \rrbracket=\llbracket \mathrm{N} \rrbracket \times \llbracket \mathrm{N}^{\prime} \rrbracket$,

- $\llbracket \mathrm{N} \oplus \mathrm{N}^{\prime} \rrbracket=\llbracket \mathrm{N} \rrbracket+\llbracket \mathrm{N}^{\prime} \rrbracket$, 
- $\llbracket \mathrm{N} \otimes \mathrm{N}^{\prime} \rrbracket=\llbracket \mathrm{N} \rrbracket \otimes \llbracket \mathrm{N}^{\prime} \rrbracket$,

- $\llbracket \mathrm{N} \multimap \mathrm{N}^{\prime} \rrbracket=\llbracket \mathrm{N} \rrbracket \multimap \llbracket \mathrm{N}^{\prime} \rrbracket$,

- $\llbracket ! \mathrm{N} \rrbracket=! \llbracket \mathrm{N} \rrbracket$ and

- $\llbracket \mathrm{N}\left\lceil_{s} \rrbracket=\llbracket \mathrm{N} \rrbracket \Gamma_{\tau(s)}\right.$

With the exception of restriction, our language contains just the connectives of intuitionistic linear logic (LIL). Therefore, the evident choice is to use the following rules of intuitionistic linear logic in order to derive net refinements.

$$
\begin{aligned}
& \overline{\mathrm{N} \vdash \mathrm{N}}(\mathrm{Id}) \quad \frac{\Gamma \vdash \mathrm{N}, \Delta \vdash \mathrm{N}^{\prime}}{\Gamma, \Delta \vdash \mathrm{N}^{\prime}}(\mathrm{Cut}) \quad \frac{\Gamma, \mathrm{N}, \mathrm{N}^{\prime} \vdash \mathrm{N}^{\prime \prime}}{\Gamma, \mathrm{N}^{\prime}, \mathrm{N} \vdash \mathrm{N}^{\prime \prime}}(\mathrm{Ex}) \\
& \overline{\Gamma, \mathbf{0} \vdash \mathrm{N}}(\mathbf{0} \vdash) \quad \overline{\Gamma \vdash \mathbf{1}}(\vdash \mathbf{1}) \quad \frac{\Gamma \vdash \mathrm{N}}{\Gamma, \mathrm{I} \vdash \mathrm{N}}(\mathrm{I} \vdash) \quad \overline{\vdash \mathrm{I}}(\vdash \mathrm{I}) \\
& \frac{\Gamma \vdash \mathrm{N} \Delta \vdash \mathrm{N}^{\prime}}{\Gamma, \Delta \vdash \mathrm{N} \otimes \mathrm{N}^{\prime}}(\vdash \otimes) \quad \frac{\Gamma, \mathrm{N}, \mathrm{N}^{\prime} \vdash \mathrm{N}^{\prime \prime}}{\Gamma, \mathrm{N} \otimes \mathrm{N}^{\prime} \vdash \mathrm{N}^{\prime \prime}}(\otimes \vdash) \\
& \frac{\Gamma \vdash N}{\Gamma \vdash N \oplus N^{\prime}}(\vdash \oplus \mathrm{l}) \quad \frac{\Gamma \vdash N^{\prime}}{\Gamma \vdash \mathrm{N} \oplus \mathrm{N}^{\prime}}(\otimes \vdash \mathrm{r}) \\
& \frac{\Gamma, \mathrm{N} \vdash \mathrm{N}^{\prime \prime} \Gamma, \mathrm{N}^{\prime} \vdash \mathrm{N}^{\prime \prime}}{\Gamma, \mathrm{N} \oplus \mathrm{N}^{\prime} \vdash \mathrm{N}^{\prime \prime}}(\otimes \vdash) \quad \frac{\Gamma, \mathrm{N} \Gamma \vdash \mathrm{N}^{\prime}}{\Gamma \vdash \mathrm{N} \wedge \mathrm{N}^{\prime}}(\vdash \wedge) \\
& \frac{\Gamma, \mathrm{N} \vdash \mathrm{N}^{\prime \prime}}{\Gamma, \mathrm{N} \wedge \mathrm{N}^{\prime} \vdash \mathrm{N}^{\prime \prime}}(\mathrm{l} \wedge \vdash) \quad \frac{\Gamma, \mathrm{N}^{\prime} \vdash \mathrm{N}^{\prime \prime}}{\Gamma, \mathrm{N} \wedge \mathrm{N}^{\prime} \vdash \mathrm{N}^{\prime \prime}}(\mathrm{r} \wedge \vdash) \\
& \frac{\Gamma \vdash \mathrm{N} \mathrm{N}^{\prime} \vdash \mathrm{N}^{\prime \prime}}{\Gamma, \mathrm{N} \multimap \mathrm{N}^{\prime} \vdash \mathrm{N}^{\prime \prime}}(\multimap \vdash) \quad \frac{\Gamma, \mathrm{N} \vdash \mathrm{N}^{\prime}}{\Gamma \vdash \mathrm{N} \multimap \mathrm{N}^{\prime}}(\vdash \multimap)
\end{aligned}
$$




$$
\overline{! \mathrm{N} \vdash \mathrm{I} \wedge \mathrm{N} \wedge(! \mathrm{N} \otimes ! \mathrm{N})}(! \multimap)
$$

The following result, which is an immediate consequence of Theorem 7.3, is our justification for using this as a basis of a proof system for nets.

Proposition 9.1 Let $\llbracket-\rrbracket$ be an interpretation of $\mathcal{N}$ in GNet. If $\Gamma \vdash \mathrm{N}$ then there is a morphism from $\llbracket \Gamma \rrbracket$ to $\llbracket \mathrm{N} \rrbracket$.

The existence of a derivation $\mathrm{N} \vdash \mathrm{N}^{\prime}$ implies the existence of a morphism from $\llbracket \mathrm{N} \rrbracket$ to $\llbracket \mathrm{N}^{\prime} \rrbracket$ in GNet, and hence that $\mathrm{N}^{\prime}$ simulates $\mathrm{N}$.

We would like to extend our system to deal with restriction by adding a rule of the form:

$$
\frac{\mathrm{N} \vdash \mathrm{N}^{\prime}}{\mathrm{N}\left\lceil{ } _ { s } \vdash \mathrm { N } ^ { \prime } \left\lceil s^{\prime}\right.\right.}
$$

together with a suitable side condition on $s$ and $s^{\prime}$. Unfortunately, it follows from Propsition 8.4 that the side condition will depend on the choice of morphism between $\mathrm{N}^{\prime}$ and $\mathrm{N}$. The derivability of $\mathrm{N} \vdash \mathrm{N}^{\prime}$ implies only that there is a morphism from $\mathrm{N}^{\prime}$ to $\mathrm{N}$.

For this reason, it seems likely that it would be profitable to develop a proof system based on Girard and Lafont's term assignment system for intuitionistic linear logic [GL87], in which one derives sequents of the form $x: \Gamma \vdash t: A$. Our rule for restriction would then be of the form:

$$
\frac{x: \mathrm{N} \vdash t: \mathrm{N}^{\prime}}{x: \mathrm{N} \Gamma_{s} \vdash t: \mathrm{N}^{\prime} \Gamma_{s^{\prime}}}
$$

with the side condition that $s \leq s^{\prime} t$.

This appears to be a promising direction for future investigation, and is currently work in progress.

\section{Marked Nets}

In this paper, as in [Bro90, BG90, BG], we have defined categories of nets without an initial marking. Other authors ([Win87], [MM88a]) have constructed categories of marked nets. An important consequence of the results 
of Section 6 is that they provide a clear indication as to how to extend our work to construct a category of marked nets.

Lemma 10.1 The following data:

- objects: marked nets $\langle\mathrm{N}, M\rangle$,

- morphisms: pairs $\langle f, F\rangle$ such that $\langle f, F\rangle$ is a morphism from $\mathrm{N}$ to $\mathrm{N}^{\prime}$ in GNet and $\left\langle M, M^{\prime}\right\rangle$ is $F$-ok, and

- composition: as in GNet

define a category, MNet.

Proof: It is clear that if $\langle\mathrm{N}, M\rangle$ is a marked net then $\langle M, M\rangle$ is $i d$-ok and so we have identities. Composition is well-defined since if $\left\langle M_{0}, M_{1}\right\rangle$ is $F$-ok and $\left\langle M_{1}, M_{2}\right\rangle$ is $G$-ok then $M_{0} F \leq M_{1}$ and $M_{1} G \leq M_{2}$ and so $M_{0} F G \leq M_{2}$ whence $\left\langle M_{0}, M_{1}\right\rangle$ is $F G$-ok. Associativity is inherited from GNet.

There is an evident forgetful functor $\mathcal{U}:$ MNet $\rightarrow$ GNet mapping a marked net $\langle\mathrm{N}, M\rangle$ to $\mathrm{N}$ and a morphism $\langle f, F\rangle$ to $\langle f, F\rangle$.

Lemma 10.2 The assignment:

$$
\mathrm{N} \longmapsto\langle\mathrm{N}, 0\rangle \quad \text { and } \quad\langle f, F\rangle \longmapsto\langle f, F\rangle
$$

where 0 is the constant zero function, defines a full and faithful functor $\mathcal{F}$ :

GNet $\rightarrow$ MNet.

Proof: $\mathcal{F}$ is well-defined since $\langle 0,0\rangle$ is $F$-ok for any $F$. $\mathcal{F}$ is clearly faithful, and is full since every map from $\langle\mathrm{N}, 0\rangle$ to $\left\langle\mathrm{N}^{\prime}, 0\right\rangle$ in MNet is a map from $\mathrm{N}$ to $\mathrm{N}^{\prime}$ in GNet.

Proposition $10.3 \mathcal{F}$ is left adjoint to $\mathcal{U}$ 

Proof: $\operatorname{MNet}\left(\mathcal{F N},\left\langle\mathrm{N}^{\prime}, M^{\prime}\right\rangle\right)=\operatorname{MNet}\left(\langle\mathrm{N}, 0\rangle,\left\langle\mathrm{N}^{\prime}, M^{\prime}\right\rangle\right)$
$=\left\{\langle f, F\rangle \in \operatorname{GNet}\left(\mathrm{N}, \mathrm{N}^{\prime}\right) \mid\left\langle 0, M^{\prime}\right\rangle\right.$ is $F$-ok $\}$$$
=\operatorname{GNet}\left(\mathrm{N}, \mathrm{N}^{\prime}\right) \text { since }\left\langle 0, M^{\prime}\right\rangle
$$$$
\text { is } F \text {-ok for any } F, M^{\prime}
$$$$
=\operatorname{GNet}\left(\mathrm{N}, \mathcal{U}\left\langle\mathrm{N}^{\prime}, M^{\prime}\right\rangle\right) \text {. }
$$

as required.

If we extend the definition of a marking to a function $M: B \rightarrow \mathbb{N} \cup\{\omega\}$ then we can also construct a right adjoint to $\mathcal{U}$.

Lemma 10.4 The assignment:

$$
\mathrm{N} \longmapsto\langle\mathrm{N}, \omega\rangle \text { and }\langle f, F\rangle \longmapsto\langle f, F\rangle,
$$

where $\omega$ is the constant $\omega$ function, defines a full and faithful functor $\mathcal{G}$ : GNet $\rightarrow$ MNet.

Proof: $\mathcal{G}$ is well-defined since $\langle\omega, \omega\rangle$ is $F$-ok for any $F . \mathcal{G}$ is clearly faithful, and is full since every map from $\langle\mathrm{N}, \omega\rangle$ to $\left\langle\mathrm{N}^{\prime}, \omega\right\rangle$ in MNet is a map from $\mathrm{N}$ to $\mathrm{N}^{\prime}$ in GNet.

Proposition $10.5 \mathcal{G}$ is right adjoint to $\mathcal{U}$.

Proof: $\operatorname{MNet}\left(\langle\mathrm{N}, M\rangle, \mathcal{G} \mathrm{N}^{\prime}\right)=\operatorname{MNet}(\langle\mathrm{N}, M\rangle,\langle\mathrm{N}, \omega\rangle)$

$$
\begin{aligned}
= & \left\{\langle f, F\rangle \in \operatorname{GNet}\left(\mathrm{N}, \mathrm{N}^{\prime}\right) \mid\langle M, \omega\rangle\right. \\
& \text { is } F \text {-ok }\} \\
= & \operatorname{GNet}\left(\mathrm{N}, \mathrm{N}^{\prime}\right) \text { since }\langle M, \omega\rangle \\
& \text { is } F \text {-ok for any } F, M^{\prime} \\
= & \operatorname{GNet}\left(\mathcal{U}\left\langle\mathrm{N}^{\prime}, M^{\prime}\right\rangle, \mathrm{N}^{\prime}\right) .
\end{aligned}
$$

as required.

Corollary 10.6 GNet is isomorphic to both a full reflective and a full coreflective subcategory of MNet.

Applying the adjoint functor theorem ${ }^{5}$ shows that $\mathcal{U}$ preserves any (small)

\footnotetext{
${ }^{5}$ The solution set condition is trivial since any morphism $\langle f, F\rangle: \mathrm{N} \rightarrow \mathcal{U} \mathrm{N}^{\prime}$ equals $\mathcal{U}\left(\langle f, F\rangle: \mathcal{F N} \rightarrow \mathrm{N}^{\prime}\right)$.
} 
limits and colimits that exist in MNet. Thus, limits and colimits in MNet can differ from those in GNet only in their action on markings. In particular, we have the following results.

Proposition 10.7 MNet has binary products given by,

$$
\left\langle\mathrm{N}_{0}, M_{0}\right\rangle \times\left\langle\mathrm{N}_{1}, M_{1}\right\rangle=\left\langle\mathrm{N}_{0} \times \mathrm{N}_{1}, M_{0} \wedge M_{1}\right\rangle
$$

where $\mathrm{N}_{0} \times \mathrm{N}_{1}$ is the product of $\mathrm{N}_{0}$ and $\mathrm{N}_{1}$ in GNet, and $M_{0} \wedge M_{1}: B_{0}+B_{1} \rightarrow$ $\mathbb{N}$ is given by $M_{0} \wedge M_{1}\left(i n_{i}(b)\right)=M_{i}(b)$.

Proof: Projections in GNet are given by $\left\langle\pi_{i}, i n_{i}\right\rangle:\left\langle\mathrm{N}_{0} \times \mathrm{N}_{1}\right\rangle \rightarrow$ $\mathrm{N}_{i} .\left\langle\pi_{i}, i n_{i}\right\rangle$ is a morphism from $\left\langle\mathrm{N}_{0}, M_{0}\right\rangle \times\left\langle\mathrm{N}_{1}, M_{1}\right\rangle$ to $\left\langle\mathrm{N}_{i}, M_{i}\right\rangle$ as $\left(\left\langle M_{0} \wedge M_{1}\right\rangle\right) i n_{i}=M_{i} \leq M_{i}$ and so $\left\langle M_{0} \wedge M_{1}, M_{i}\right\rangle$ is $i n_{i}$-ok.

Now suppose that there exists $\left\langle f_{i}, F_{i}\right\rangle:\langle\mathrm{N}, M\rangle \rightarrow\left\langle\mathrm{N}_{i}, M_{i}\right\rangle$. Then there exists a unique map $\left\langle\left\langle f_{0}, f_{1}\right\rangle, F_{0}+F_{1}\right\rangle: \mathrm{N} \rightarrow \mathrm{N}_{0} \times \mathrm{N}_{1}$ in GNet such that $\left\langle\pi_{i}, i n_{i}\right\rangle\left\langle\left\langle f_{0}, f_{1}\right\rangle, F_{0}+F_{1}\right\rangle=\left\langle f_{i}, F_{i}\right\rangle$.

However, the $\left\langle f_{i}, F_{i}\right\rangle$ are morphisms in MNet and so each $\left\langle M, M_{i}\right\rangle$ is $F_{i}$-ok. Therefore, $M\left(F_{0}+F_{1}\right)\left(i n_{i}(b)\right)=M F_{i}(b) \leq M_{i}(b)=$ $\left(M_{0} \wedge M_{1}\right) i n_{i}(b)$ and so $\left\langle M, M_{0} \wedge M_{1}\right\rangle$ is $\left(F_{0}+F_{1}\right)$-ok whence $\left\langle\left\langle f_{0}, f_{1}\right\rangle, F_{0}+F_{1}\right\rangle$ is a morphism in MNet. This completes the proof.

Proposition 10.8 MNet has binary coproducts given by,

$$
\left\langle\mathrm{N}_{0}, M_{0}\right\rangle+\left\langle\mathrm{N}_{1}, M_{1}\right\rangle=\left\langle\mathrm{N}_{0}+\mathrm{N}_{1}, M_{0} \oplus M_{1}\right\rangle
$$

where $\mathrm{N}_{0}+\mathrm{N}_{1}$ is the coproduct of $\mathrm{N}_{0}$ and $\mathrm{N}_{1}$ in GNet, and $M_{0} \oplus M_{1}$ : $B_{0} \times B_{1} \rightarrow \mathbb{N}$ is given by $M_{0} \oplus M_{1}\left\langle b_{0}, b_{1}\right\rangle=\max \left\{M_{0}\left(b_{0}\right), M_{1}\left(b_{1}\right)\right\}$.

Proof: Injections in GNet are given by $\left\langle i n_{i}, \pi_{i}\right\rangle: \mathrm{N}_{i} \rightarrow \mathrm{N}_{0}+\mathrm{N}_{1}$. $\left\langle i n_{i}, \pi_{i}\right\rangle$ is a morphism from $\left\langle\mathrm{N}_{i}, M_{i}\right\rangle$ to $\left\langle\mathrm{N}_{0}, M_{0}\right\rangle+\left\langle\mathrm{N}_{1}, M_{1}\right\rangle$ as $M_{0} \oplus M_{1}\left(\left\langle b_{0}, b_{1}\right\rangle\right)=\max \left\{M_{0}\left(b_{0}\right), M_{1}\left(b_{1}\right)\right\} \geq M_{i}\left(b_{i}\right)=M_{i} \pi_{i}\left(\left\langle b_{0}, b_{1}\right\rangle\right)$ and so $\left\langle M_{i}, M_{0} \oplus M_{1}\right\rangle$ is $\pi_{i}$-ok.

Now suppose that there exists $\left\langle f_{i}, F_{i}\right\rangle:\left\langle\mathrm{N}_{i}, M_{i}\right\rangle \rightarrow\langle\mathrm{N}, M\rangle$. Then there exists a unique map $\left\langle f_{0}+f_{1},\left\langle F_{0}, F_{1}\right\rangle\right\rangle: \mathrm{N}_{0}+\mathrm{N}_{1} \rightarrow \mathrm{N}$ in GNet such that $\left\langle f_{0}+f_{1},\left\langle F_{0}, F_{1}\right\rangle\right\rangle\left\langle i n_{i}, \pi_{i}\right\rangle=\left\langle f_{i}, F_{i}\right\rangle$.

However, the $\left\langle f_{i}, F_{i}\right\rangle$ are morphisms in MNet and so each $\left\langle M_{i}, M\right\rangle$ 
is $F_{i}$-ok. Therefore, $M_{0} \oplus M_{1}\left\langle F_{0}, F_{1}\right\rangle(b)=M_{0} \oplus M_{1}\left\langle F_{0} b, F_{1} b\right\rangle=$ $\max \left\{M_{0} F_{0} b, M_{1} F_{1} b\right\} \leq M(b)$ as $M(b)$ is greater than each $M_{i} F_{i}(b)$ since, each $\left\langle M_{i}, M\right\rangle$ is $F$-ok. Thus $\left\langle f_{0}+f_{1},\left\langle F_{0}, F_{1}\right\rangle\right\rangle$ is a morphism in MNet. This completes the proof.

Proposition 10.9 MNet has initial and terminal objects given by,

$$
0_{\mathrm{MNet}}=\left\langle 0_{\mathrm{GNet}}, 0\right\rangle \quad \text { and } \quad 1_{\mathrm{MNet}}=\left\langle 1_{\mathrm{GNet}}, \emptyset\right\rangle .
$$

Proof: There exists a unique $*$ from 0 to $\mathrm{N}$ in GNet. This is also a map from 0 to $\langle\mathrm{N}, M\rangle$ in MNet for any $M$ since $M \geq 0 *=0$. There exists a unique $*$ from $\mathrm{N}$ to 1 in GNet. This is also a map from $\langle\mathrm{N}, M\rangle$ to 1 in MNet for any $M$ since $\emptyset \geq M \emptyset=\emptyset$.

The symmetric monoidal closed structure of GNet does not lift to MNet as smoothly as we might have hoped. Recall from Section 8 that the net $\mathrm{N}_{0} \otimes \mathrm{N}_{1}$ is given by,

$$
\left\langle E_{0} \times E_{1}, B_{0}^{E_{1}} \times B_{1}^{E_{0}}, \text { pre }_{0} \otimes \text { pre }_{1}, \text { post }_{0} \otimes \text { post }_{1}\right\rangle .
$$

Therefore, a marking of the tensor product of marked nets $\left\langle\mathrm{N}_{0}, M_{0}\right\rangle$ and $\left\langle\mathrm{N}_{1}, M_{1}\right\rangle$ is a function $M_{0} \otimes M_{1}$ from $B_{0}^{E_{1}} \times B_{1}^{E_{0}}$ to $\mathbb{N}$. The natural choice appears to be to define,

$$
M_{0} \otimes M_{1}\langle f, g\rangle=\max _{E_{0}}\left\{M_{1} f\left(e_{0}\right)\right\}+\max _{E_{1}}\left\{M_{0} g\left(e_{1}\right)\right\} .
$$

This has the appealing property that if $M_{0} \downarrow e_{0}$ and $M_{1} \downarrow e_{1}$ then $M_{0} \otimes M_{1} \downarrow$ $\left\langle e_{0}, e_{1}\right\rangle$. Unfortunately, with this definition of $M_{0} \otimes M_{1}$, it is not possible to give a definition of $M_{0} \multimap M_{1}$ in such a way that $-\otimes\left\langle\mathrm{N}_{0}, M_{0}\right\rangle$ is left adjoint to $-\multimap\left\langle\mathrm{N}_{0}, M_{0}\right\rangle$. The difficulty is that a marking of the net $\mathrm{N}_{0} \multimap \mathrm{N}_{1}$ which is,

$$
\left\langle E_{1}^{E_{0}} \times B_{0}^{B_{1}}, E_{0} \times B_{1}, \text { pre }_{0} \multimap \text { pre }_{1}, \text { post }_{0} \multimap \text { post }_{1}\right\rangle .
$$

is a function $M_{0} \multimap M_{1}$ from $E_{0} \times B_{1}$ to $\mathbb{N}$. For the adjunction we require,

$$
\begin{aligned}
\forall b_{2} \in B_{2} . M_{2}\left(b_{2}\right) \geq M_{0} \otimes M_{1}\langle f, g\rangle \text { iff } \quad & \forall b_{2} \in B_{2} \forall e_{1} \in E_{1} . \\
& M_{1} \multimap M_{2}\left\langle e_{1}, b_{2}\right\rangle \geq M_{0}\left(f\left(e_{1}\right)\right) .
\end{aligned}
$$


As $g$ occurs free on the left hand side of this equivalence and does not occur on the right, the definition of $M_{0} \otimes M_{1}$ can not depend on $g$. In fact, if we ignore $g$ and define,

$$
M_{0} \otimes M_{1}\langle f, g\rangle=\max _{E_{0}}\left\{M_{1} f\left(e_{0}\right)\right\} .
$$

The we can recover the closed structure by defining,

$$
M_{0} \multimap M_{1}\left\langle e_{1}, b_{0}\right\rangle=M_{1}\left(b_{1}\right)
$$

in which case $-\otimes\langle\mathrm{N}, M\rangle$ is left adjoint to $-\multimap\langle\mathrm{N}, M\rangle$. However, this is unsatisfactory as it destroys the symmetry of $\otimes$ and has no natural computational interpretation. These difficulties have led us to explore other possible closed structures on GNet and MNet such as those described in [LS91]. This is work in progress.

\section{A Structure in $\mathrm{M}_{\mathbb{N}}$ Set}

\section{A.1 Products and Coproducts in $\mathrm{M}_{\mathbb{N}}$ Set}

The products and coproducts in $\mathbf{M}_{\mathbb{N}}$ Set are defined in terms of the product and coproduct in Set (observe that, since products and coproducts in Set are assigned, they are also assigned in $\mathbf{M}_{\mathbb{N}} \mathbf{S e t}$ ). It is routine to prove the following lemmas.

Lemma A.1 The product of two objects $\left\langle E_{0}, B_{0}, \alpha_{0}\right\rangle$ and $\left\langle E_{1}, B_{1}, \alpha_{1}\right\rangle$ of $\mathbf{M}_{\mathbb{N}}$ Set is $\left\langle E_{0} \times E_{1}, B_{0}+B_{1}, \alpha_{0} \wedge \alpha_{1}\right\rangle$, where the function $\alpha_{0} \wedge \alpha_{1}$ from $\left(E_{0} \times E_{1}\right) \times\left(B_{0}+B_{1}\right)$ into $\mathbb{N}$ is given by

$$
\left(\alpha_{0} \wedge \alpha_{1}\right)\left\langle e_{0}, e_{1}, b\right\rangle= \begin{cases}\alpha_{0}\left\langle e_{0}, b_{0}\right\rangle & \text { if } b=\left(b_{0}, 0\right) \\ \alpha_{1}\left\langle e_{1}, b_{1}\right\rangle & \text { if } b=\left(b_{1}, 1\right) .\end{cases}
$$

The projection from $\left\langle E_{0} \times E_{1}, B_{0}+B_{1}, \alpha_{0} \wedge \alpha_{1}\right\rangle$ to $\left\langle E_{i}, B_{i}, \alpha_{i}\right\rangle$ is the morphism $\left\langle\pi_{i}, i n_{i}\right\rangle$, where $\pi_{i}$ is the $i$ th projection in Set and $i n_{i}$ the $i$ th canonical injection in Set.

Lemma A.2 The coproduct of two objects $\left\langle E_{0}, B_{0}, \alpha_{0}\right\rangle$ and $\left\langle E_{1}, B_{1}, \alpha_{1}\right\rangle$ 
of $\mathbf{M}_{\mathbb{N}}$ Set is $\left\langle E_{0}+E_{1}, B_{0} \times B_{1}, \alpha_{0} \oplus \alpha_{1}\right\rangle$ where the function $\alpha_{0} \oplus \alpha_{1}$ from $\left(E_{0}+E_{1}\right) \times\left(B_{0} \times B_{1}\right)$ into $\mathbb{N}$ is given by

$$
\left(\alpha_{0} \oplus \alpha_{1}\right)\left\langle e, b_{0}, b_{1}\right\rangle= \begin{cases}\alpha_{0}\left\langle e_{0}, b_{0}\right\rangle & \text { if } e=\left(e_{0}, 0\right) \\ \alpha_{1}\left\langle e_{1}, b_{1}\right\rangle & \text { if } e=\left(e_{1}, 1\right) .\end{cases}
$$

The injection from $\left\langle E_{i}, B_{i}, \alpha_{i}\right\rangle$ into $\left\langle E_{0}+E_{1}, B_{0} \times B_{1}, \alpha_{0} \oplus \alpha_{1}\right\rangle$ is the morphism $\left\langle\pi_{i}, i n_{i}\right\rangle$.

\section{A.2 A Monoidal Closed Structure on $\mathrm{M}_{\mathbb{N}}$ Set}

Lemma A.3 The functor $\otimes: \mathbf{M}_{\mathbb{N}}$ Set $\times \mathbf{M}_{\mathbb{N}}$ Set $\rightarrow \mathbf{M}_{\mathbb{N}}$ Set which takes the pair $\left\langle\left\langle E_{0}, B_{0}, \alpha_{0}\right\rangle,\left\langle E_{1}, B_{1}, \alpha_{1}\right\rangle\right\rangle$ of objects of $\mathbf{M}_{\mathbb{N}}$ Set to

$$
\left\langle E_{0} \times E_{1}, B_{0}^{E_{1}} \times B_{1}^{E_{0}}, \alpha_{0} \otimes \alpha_{1}\right\rangle,
$$

where $\left(\alpha_{0} \otimes \alpha_{1}\right)\left\langle e_{0}, e_{1}, f, g\right\rangle=\alpha_{0}\left\langle e_{0}, f e_{1}\right\rangle+\alpha_{1}\left\langle e_{1}, g e_{0}\right\rangle$, gives rise to a symmetric monoidal structure on $\mathbf{M}_{\mathbb{N}}$ Set (also denoted $\otimes$ ) with unit $I=\langle\{*\},\{*\}, 0\rangle$.

Lemma A.4 The functor $\left(-\otimes\left\langle E_{0}, B_{0}, \alpha_{0}\right\rangle\right)$ has a right adjoint $(-\multimap$ $\left.\left\langle E_{0}, B_{0}, \alpha_{0}\right\rangle\right)$ which takes the object $\left\langle E_{1}, B_{1}, \alpha_{1}\right\rangle$ of $\mathbf{M}_{\mathbb{N}}$ Set to the object

$$
\left\langle E_{1}^{E_{0}} \times B_{0}^{B_{1}}, E_{0} \times B_{1}, \alpha_{0} \multimap \alpha_{1}\right\rangle,
$$

where $\left(\alpha_{0} \multimap \alpha_{1}\right)\left\langle f, F, e_{0}, b_{1}\right\rangle=\alpha_{1}\left(f e_{0}, b_{1} \ominus \alpha_{0}\left(e_{0}, F b_{1}\right)\right.$.

The symbol $\ominus$ represents truncated subtraction, which coincides with natural number subtraction unless its first argument is smaller than its second, in which case it evaluates to 0 . This is analogous to a construction used by Lawvere on the positive reals, see [Law73].

\section{B Products in GNet}

Recall, from Section 6, that we identify a Petri net $\langle E, B$, pre, post $\rangle$ with the object $\left\langle\langle E, B\right.$, pre $\rangle,\left\langle B, E\right.$, post $\left.\left.{ }^{o p}\right\rangle\right\rangle$ of $\mathbf{M}_{\mathbb{N}}$ Set $\times \mathbf{M}_{\mathbb{N}}$ Set $^{*}$.

It follows from Theorem 7.3 that the product of two nets $\left\langle E_{0}, B_{0}\right.$, pre $_{0}$, post $\left._{0}\right\rangle$ and $\left\langle E_{1}, B_{1}\right.$, pre $_{1}$, post $\left._{1}\right\rangle$ in GNet is given by the product of $\left\langle\left\langle E_{0}, B_{0}\right.\right.$, pre $\left._{0}\right\rangle$, $\left\langle B_{0}, E_{0}\right.$, post $\left.\left._{0}^{o p}\right\rangle\right\rangle$ and $\left\langle\left\langle E_{1}, B_{1}\right.\right.$, pre $\left._{1}\right\rangle,\left\langle B_{1}, E_{1}\right.$, post $\left.\left._{1}^{o p}\right\rangle\right\rangle$ in $\mathbf{M}_{\mathbb{N}}$ Set $\times \mathbf{M}_{\mathbb{N}}$ Set $^{*}$. 
The product of two objects $\left\langle E_{0}, B_{0}, \alpha_{0}\right\rangle$ and $\left\langle E_{1}, B_{1}, \alpha_{1}\right\rangle$ in $\mathbf{M}_{\mathbb{N}}$ Set is the object $\left\langle E_{0} \times E_{1}, B_{0}+B_{1}, \alpha_{0} \wedge \alpha_{1}\right\rangle$ where

$$
\left(\alpha_{0} \wedge \alpha_{1}\right)\left\langle e_{0}, e_{1}, b\right\rangle= \begin{cases}\alpha_{0}\left\langle e_{0}, b_{0}\right\rangle & \text { if } b=\left(b_{0}, 0\right) \\ \alpha_{1}\left\langle e_{1}, b_{1}\right\rangle & \text { if } b=\left(b_{1}, 1\right) .\end{cases}
$$

The details are contained in [DP91]. Clearly, the product of two objects $\left\langle B_{0}, E_{0}, \alpha_{0}\right\rangle$ and $\left\langle B_{1}, E_{1}, \alpha_{1}\right\rangle$ in $\mathbf{M}_{\mathbb{N}}$ Set $^{*}$ can be defined in terms of the product in $\mathbf{M}_{\mathbb{N}}$ Set as,

$$
\begin{aligned}
\iota^{-1}\left(\iota\left(\left\langle B_{0}, E_{0}, \alpha_{0}\right\rangle\right) \times \iota\left(\left\langle B_{1}, E_{1}, \alpha_{1}\right\rangle\right)\right) & =\iota^{-1}\left(\left\langle E_{0}, B_{0}, \alpha_{0}^{o p}\right\rangle \times\left\langle E_{1}, B_{1}, \alpha_{1}^{o p}\right\rangle\right) \\
& =\iota^{-1}\left(\left\langle E_{0} \times E_{1}, B_{0}+B_{1}, \alpha_{0}^{o p} \wedge \alpha_{1}^{o p}\right\rangle\right) \\
& =\left\langle B_{0}+B_{1}, E_{0} \times E_{1},\left(\alpha_{0}^{o p} \wedge \alpha_{1}^{o p}\right)^{o p}\right\rangle \\
& =\left\langle B_{0}+B_{1}, E_{0} \times E_{1}, \alpha_{0} \wedge \alpha_{1}\right\rangle .
\end{aligned}
$$

Thus the product of $\left\langle\left\langle E_{0}, B_{0}\right.\right.$, pre $\left._{0}\right\rangle,\left\langle B_{0}, E_{0}\right.$, post $\left.\left._{0}^{\text {op }}\right\rangle\right\rangle$ and $\left\langle\left\langle E_{1}, B_{1}\right.\right.$, pre $\left._{1}\right\rangle$, $\left\langle B_{1}, E_{1}\right.$, post $\left.\left._{1}^{o p}\right\rangle\right\rangle$ in $\mathbf{M}_{\mathbb{N}}$ Set $\times \mathbf{M}_{\mathbb{N}}$ Set $^{*}$ is given by,

$$
\begin{aligned}
& \left\langle\left\langle E_{0}, B_{0}, \text { pre }_{0}\right\rangle,\left\langle B_{0}, E_{0}, \text { post }_{0}^{o p}\right\rangle\right\rangle \times\left\langle\left\langle E_{1}, B_{1}, \text { pre }_{1}\right\rangle,\left\langle B_{1}, E_{1}, \text { post }_{1}^{o p}\right\rangle\right\rangle \\
& =\left\langle\left\langle E_{0}, B_{0}, \text { pre }_{0}\right\rangle \times\left\langle E_{1}, B_{1}, \text { pre }_{1}\right\rangle\right\rangle,\left\langle B_{0}, E_{0}, \text { post }_{0}^{o p}\right\rangle \times\left\langle B_{1}, E_{1}, \text { post }_{1}^{o p}\right\rangle \\
& =\left\langle\left\langle E_{0} \times E_{1}, B_{0}+B_{1}, \text { pre }_{0} \wedge \text { pre }_{1}\right\rangle\right\rangle,\left\langle B_{0}+B_{1}, E_{0} \times E_{1}, \text { post }_{0}^{o p} \wedge \text { post }_{1}^{o p}\right\rangle \\
& =\left\langle\left\langle E_{0} \times E_{1}, B_{0}+B_{1}, \text { pre }_{0} \wedge \text { pre }_{1}\right\rangle\right\rangle,\left\langle B_{0}+B_{1}, E_{0} \times E_{1},\left(\text { post }_{0} \wedge \text { post }_{1}\right)^{o p}\right\rangle
\end{aligned}
$$

which we identify with the net $\left\langle E_{0} \times E_{1}, B_{0}+B_{1}\right.$, pre $_{0} \wedge$ pre $_{1}$, post $_{0} \wedge$ post $\left._{1}\right\rangle$.

Coproducts and the symmetric monoidal closed structure of $\mathbf{M}_{\mathbb{N}}$ Set lift to GNet in an entirely similar fashion.

\section{References}

[Amb91] S. J. Ambler. First Order Linear Logic in Symmetric Monoidal Closed Categories, PhD thesis, University of Edinburgh, 1991.

[AS79] J. R. Abrial and S. A. Schuman. Non-deteministic system specification, in G, Kahn, editor, Semantics of Concurrent Computation. Springer-Verlag, LNCS 70, 1979. 
[Bar79] M. Barr. *-Autonomous Categories, LNM, Springer-Verlag, 1979.

[BG] C. T. Brown and D. J. Gurr. A categorical linear framework for petri nets, to appear in Information and Computation.

[BG90] C. T. Brown and D. J. Gurr. A categorical linear framework for petri nets, in Proc. LICS, 1990.

[Bro90] C. T. Brown. Linear Logic and Petri Nets: Categories, Algebra and Proof, PhD thesis, University of Edinburgh, 1990.

[BG80] R. M. Burstall and J. A. Goguen. The semantics of clear, a specification language, in Proc. of Advance Course on Abstract Software Specifications, pages 292-332. Springer-Verlag, LNCS 86, 1980.

[DMM89] P. Degano, J. Meseguer, and U. Montanari. Axiomatising net computations and processes, in Proc LICS, 1989.

[DP88] V. C. V. De Paiva. The Dialectica Categories, PhD thesis, University of Cambridge, 1988.

[DP89] V. C. V. De Paiva. A dialectica-like model of linear logic, in D.H. Pitt, D.E. Rydeheard, P. Dybjer, A.M. Pitts, and A. Poigné, editors, Proc. Category Theory and Computes Science, Manchester. Springer Verlag (LNCS 389), 1989.

[DP91] V. C. V. De Paiva. A category of multirelations, Technical report number 255, Univ. of Cambridge, 1991.

[Fou80] M. P. Fourman. The logic of topoi, in J. Barwise, editor, the Handbook of Mathematical Logic.

[Gir87] J- Y. Girard. Linear logic, TCS, 50:1-102,1987.

[GL87] J- Y. Girard and Y. Lafont. Linear logic and lazy computation, in Proc. TAPSOFT 87 (Pisa), volume 2, pages 52-66, 1987. SpringerVerlag, (LNCS 250).

[HDP90] J. M. E. Hyland and V. C. V. De Paiva. Lineales, manuscript, October 1990. 
[Jen90] K. Jensen Coloured petri nets: a high level language for system design analysis, in G. Rosenberg, editor, Proc. Advances in Petri nets, 1990. Springer-Verlag, (LNCS).

[Joh77] P. T. Johnstone. Topos Theory, Academic Press, 1977.

[Jon86] C. B. Jones. Systematic Software Development using VDM, Prentice Hall, 1986.

[Law73] F. W. Lawvere. Metric spaces, generalised logic, and closed categories, in Rend. del Seminario Matematico e Fisico di Milano, volume 43, 1973.

[LS86] J. Lambek and P. J. Scott. Introduction to higher order categorical logic, CUP, 1986.

[LS91] Y. Lafont and T. Streicher. Games semantics for linear logic, in Proc. LICS, 1991.

[Mil89] R. Milner. Communication and Concurrency, Prentice Hall, 1989.

[MM88a] J. Meseguer and U. Montanari. Petri nets are monoids: A new algebraic foundation for net theory, in Proc LICS, 1988.

[MM88b] J. Meseguer and U. Montanari. Petri nets are monoids: A new algebraic foundation for net theory, TechnicaI Report SRICSL-88-3, C.S. Lab., SRI International, January 1988.

[MOM89] N. Marti-Oliet and J. Meseguer. From petri nets to linear logic, In D.H. Pitt, D.E. Rydeheard, P. Dybjer, A.M. Pitts, and A. Poigné, editors, Proc. Category Theory and Computer Science, Manchester, 1989.

[NRT90] M. Nielsen, G. Rosenberg, and P. S. Thiagarajan. Elementary transition systems, Technical Report DAIMI PB - 310, DAIMI, Århus University, April 1990.

[NW91] M. Nielsen and G. Winskel. Concurrency, Chapter in the forthcoming Handbook of Theoretical Computer Science.

[Rei85] W. Reisig. Petri Nets: an Introduction, EATCS Monographs on Theoretical Computer Science Springer-Verlag, 1985. 
[See89] R. Seeley. Linear logic, *-autonomous categories and cofree coalgebras, in Categories in Computer Science and Logic, volume 43. AMS, 1989.

[ST88] D. Sannella and A. Tarlecki. Toward formal development of programs form algebraic specifications: implementations revisited, Acta Informatica, 25:233-281, 1988.

[Ver91] D. Verity. Enrichments, manuscript, February 1991.

[Win84] G. Winskel. Categories of models for concurrency, in S.D. Brookes, A.W. Roscoe, and G. Winskel, editors, Proc. Seminar on Semantics of Concurrency (CMU, Pittsburgh), 1984. Springer-Verlag, (LNCS 197).

[Win87] G. Winskel. Petri nets, algebras, morphisms, and compositionality, Information and Computation, 72:197-238, 1987.

[Win88] G. Winskel. A category of labelled petri nets and compositional proof system, in Proc LICS, 1988.

[Wir71] N. Wirth. Program development by stepwise refinement, Comm. ACM, 14(4):221-227, 1971. 